\title{
Intershell resistance in multiwall carbon nanotubes: A Coulomb drag study
}

\author{
Lunde, Anders Mathias; Flensborg, Karsten; Jauho, Antti-Pekka
}

Published in:

Physical Review B Condensed Matter

Link to article, DOI:

10.1103/PhysRevB.71.125408

Publication date:

2005

Document Version

Publisher's PDF, also known as Version of record

Link back to DTU Orbit

Citation $(A P A)$ :

Lunde, A. M., Flensborg, K., \& Jauho, A-P. (2005). Intershell resistance in multiwall carbon nanotubes: A Coulomb drag study. Physical Review B Condensed Matter, 71(12), 125408.

https://doi.org/10.1103/PhysRevB.71.125408

\section{General rights}

Copyright and moral rights for the publications made accessible in the public portal are retained by the authors and/or other copyright owners and it is a condition of accessing publications that users recognise and abide by the legal requirements associated with these rights.

- Users may download and print one copy of any publication from the public portal for the purpose of private study or research.

- You may not further distribute the material or use it for any profit-making activity or commercial gain

- You may freely distribute the URL identifying the publication in the public portal 


\title{
Intershell resistance in multiwall carbon nanotubes: A Coulomb drag study
}

\author{
Anders Mathias Lunde, ${ }^{1,2, *}$ Karsten Flensberg, ${ }^{1}$ and Antti-Pekka Jauho ${ }^{2}$ \\ ${ }^{1} \emptyset$ rsted Laboratory, Niels Bohr Institute, University of Copenhagen, DK-2100 Copenhagen, Denmark \\ ${ }^{2}$ MIC-Department of Micro and Nanotechnology, Technical University of Denmark, Ørsteds Plads, \\ Bldg. 345 east, DK-2800 Kgs. Lyngby, Denmark \\ (Received 5 August 2004; published 16 March 2005)
}

\begin{abstract}
We calculate the intershell resistance $R_{21}$ in a multiwall carbon nanotube as a function of temperature $T$ and Fermi level $\varepsilon_{\mathrm{F}}$ (e.g., a gate voltage), varying the chirality of the inner and outer tubes. This is done in a so-called Coulomb drag setup, where a current $I_{1}$ in one shell induces a voltage drop $V_{2}$ in another shell by the screened Coulomb interaction between the shells neglecting the intershell tunneling. We provide benchmark results for $R_{21}=V_{2} / I_{1}$ within the Fermi liquid theory using Boltzmann equations. The band structure gives rise to strongly chirality-dependent suppression effects for the Coulomb drag between different tubes due to selection rules combined with mismatching of wave vector and crystal angular momentum conservation near the Fermi level. This gives rise to orders of magnitude changes in $R_{21}$ and even the sign of $R_{21}$ can change depending on the chirality of the inner and outer tube and misalignment of inner and outer tube Fermi levels. However for any tube combination, we predict a dip (or peak) in $R_{21}$ as a function of gate voltage, since $R_{21}$ vanishes at the electron-hole symmetry point. As a by-product, we classified all metallic tubes into either zigzaglike or armchairlike, which have two different nonzero crystal angular momenta $\mathfrak{m}_{a}, \mathfrak{m}_{b}$ and only zero angular momentum, respectively.
\end{abstract}

DOI: 10.1103/PhysRevB.71.125408

PACS number(s): 73.63.Fg, 73.23.-b

\section{INTRODUCTION}

\section{A. General considerations on nanotubes}

Carbon nanotubes are widely recognized as being among the most promising materials for future nanotechnology applications. Furthermore, they are of fundamental scientific interest due to several unique electronic, mechanical, and thermal properties. ${ }^{1}$ These properties often depend on the microscopic details of their composition, e.g., the way the graphene sheets are rolled into tubes and whether one has a single or multiwall carbon nanotube or a rope or bundle of these. Electrical transport measurements have shown a tendency for ballistic transport in individual singlewall carbon nanotubes $^{2-4}$ (SWCNT) and diffusive transport in multiwall carbon nanotubes ${ }^{4-7}$ (MWCNT), but this issue is not completely settled yet ${ }^{8}$ and seems to depend crucially on the contacts to the tubes and the amount of defects and impurities in and near the tube. Many experiments ${ }^{9-15}$ have explored the Coulomb blockade regime, where the tube can be treated as a quantum dot, due to poor electric contact. More recently, better electrical contacts have been achieved, ${ }^{3,16-18}$ which gives larger conductance, approaching the predicted $4 e^{2} / h$, and a coherent (or Landauer-Büttiker-like) regime is thereby reached. Palladium seems to be a promising candidate for good future ohmic contacts. ${ }^{17,18}$ Another interesting feature of carbon nanotubes is their one-dimensional nature, which may have profound consequences on the basic physical phenomenology for their description: SWCNT's have been predicted to be Luttinger liquids ${ }^{19,20}$ and some experimental evidence exists ${ }^{21,22}$ even though other interpretations have been suggested. ${ }^{23}$ Whether MWCNT's are Fermi or Luttinger liquids has been investigated extensively experimentally ${ }^{24-26}$ and theoretically ${ }^{27}$ and seems to depend on the situation, but is still subject to debate. Also in ropes the situation is not clear yet. ${ }^{28}$

The structure of this paper is as follows. We begin by introducing the intershell resistance problem in MWCNT's and our approach to it in Sec. I B. In Sec. I C we review the basic qualitative features of our theory of the intershell resistance using a Coulomb drag setup. Sections II and III are devoted to a summary of the band structure and a calculation of the screened Coulomb matrix element including the important suppression rules for backscattering in metallic tubes, and in Sec. IV we indicate how the standard transresistance formulas are modified in the nanotube configuration. Sections V-VII give our results for several different nanotube combinations. Details of the nanotube band structure and the screening model including the band structure are found in the Appendixes.

\section{B. Intershell resistance in MWCNT's}

Let us now consider electron interaction and transport in the concentric tubes (or shells) in a MWCNT. Yoon et al. ${ }^{29}$ have argued theoretically that the intershell tunneling of electrons is vanishingly small between both commensurable and incommensurable long defect-free MWCNT's. Lack of intertube tunneling is also expected in nanotube ropes. ${ }^{30}$ Furthermore, Aharonov-Bohm experiments ${ }^{5}$ indicate that current only flows in the outer tube in a MWCNT. Another experiment by Collins et $a .^{31}$ supports this picture and finds no leakage between the shells in the low-bias limit. This is concluded by removing the shells in a MWCNT one by one and measuring the gate voltage response of the remaining MWCNT after each shell removal. Other shell removing experiments have also been reported. ${ }^{32-35}$ Furthermore, Cum- 


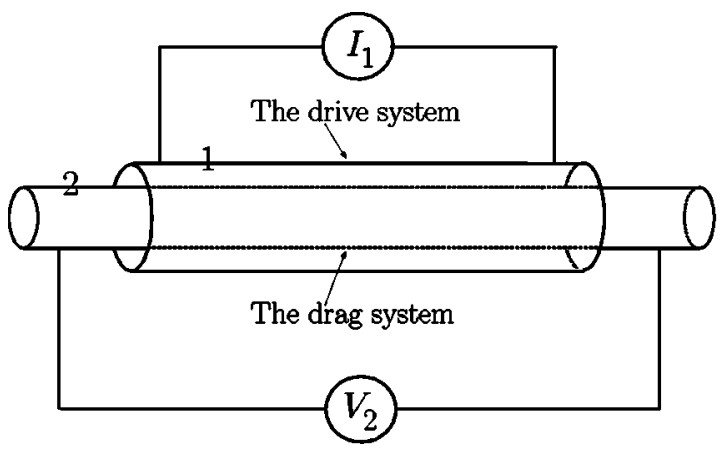

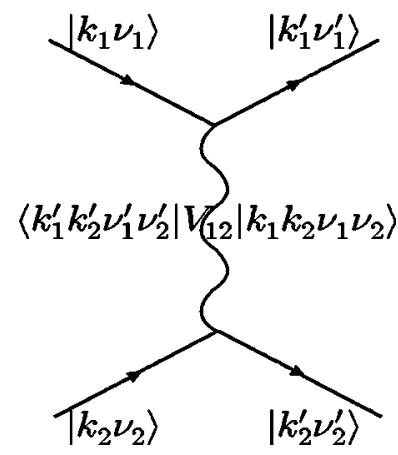

FIG. 1. (Left): The experimental setup to directly measure the Coulomb drag effect in a MWCNT. The intershell resistance is $R_{21}=V_{2} / I_{1}$. (Right): The basic mechanism in the intershell resistance in a drag configuration: the intershell $e-e$ interaction and thereby momentum transfer to induce the voltage drop $V_{2}$. ings and Zettl ${ }^{36}$ have demonstrated relative motion between the inner and outer shells in a MWCNT, indicating that the shells are weakly coupled by van der Waals forces. In addition to Yoon et al. ${ }^{29}$ also other theoretical papers have calculated the intershell resistance using tunneling as the only mechanism. ${ }^{37-45}$ For example Roche and co-workers ${ }^{37-39}$ have considered the time evolution of a wave packet initially on the outer tube in a disorder-free MWCNT including tunneling in a tight-binding approximation. This is not in contrast to Ref. 29 due to the localization of the wave packet of Refs. 37-39. ${ }^{29}$ Using density functional theory (DFT), Hansson and Stafström ${ }^{41}$ consider concentric armchair tubes and find no essential change in the conductance steps for a ballistic MWCNT, when the intershell tunneling is turned on and off. References 42 and 43 also model intershell tunneling by DFT. Very recently, experiments with a MWCNT with 11 contacts on the outer tube, where a current is driven though some of the tube and a voltage drop is measured elsewhere on the tube, have been published. ${ }^{46}$ Using a transmission line model, information about the intershell conductance is deduced.

In the present paper, we approach the intershell resistance in a MWCNT from a different point of view: We consider the intershell resistance $R_{21}$ from the electron-electron $(e-e)$ interaction between the shells neglecting tunneling, i.e., in a Coulomb drag configuration. In general, Coulomb drag ${ }^{47,48}$ means that moving charges in one subsystem (the drive subsystem) exchange momentum (and other quantum numbers) with carriers in a nearby subsystem (the probe or drag subsystem), thus exerting a drag force on the probe, inducing a current, or a voltage, in the probe (see Fig. 1). Here the intershell or transresistance $R_{21}=V_{2} / I_{1}$ is found as a function of gate voltage (i.e., Fermi level $\varepsilon_{\mathrm{F}}$ ) and temperature $T$, varying the chirality of the inner and outer tubes. Once the chiralities of the tubes are chosen, our theory has no remaining free parameters. Coulomb drag is a unique transport measurement in the sense that the $R_{21}$ is dominated by the intershell Coulomb interaction. ${ }^{49}$ Therefore serious attention to the intershell Coulomb matrix element and the use of proper Bloch states of the individual tubes is necessary. As will be seen below, the effects of including the band structure (and the underlying symmetries of the constituent nanotubes) are absolutely crucial, leading to orders-of-magnitude changes in the intershell resistance, occasionally also revers- ing its sign. Furthermore, the present work also gives a new source of friction against relative motion of concentric tubes, which could be considered in the context of using MWCNT's as GHz nanomechanical oscillators. ${ }^{50}$

A direct measurement of the intershell resistance in a Coulomb drag setup (Fig. 1) requires independent contacts on an inner and an outer tube, a difficult but possible technological achievement ${ }^{51}$ in light of the resent shell removal experiments. ${ }^{31-35}$ As a model, we consider two shells, but our considerations can be extended for many shells. Also, a direct growth of double wall tubes seems feasible. ${ }^{52}$

Coulomb drag has been an extremely successful tool in studying interactions in coupled quantum wells ${ }^{53-58}$ (notably in the quantum Hall regimes ${ }^{53,59}$ ), and indeed it was realized very early that Coulomb drag between Luttinger liquids would be an important object to study. ${ }^{60-66}$ These studies focused on Coulomb drag on either crossed or adjacent subsystems, and used very simple models for the Coulomb interaction. Several interesting theoretical predictions emerged from these papers, some of which may have been confirmed experimentally. ${ }^{67}$ We work in the Fermi liquid framework using Boltzmann equations. We think that it is important to establish a clear picture of what one expects within this simple model before turning to strongly interacting theories. Note that our approach also gives valuable information about drag between parallel tubes.

\section{Nanotube Coulomb drag-qualitative features of the theory}

As explained in detail in subsequent sections, the transresistance or intershell resistance $R_{21}$ is computed from the expression

$$
R_{21} \propto \int \sum(\mathrm{SR})^{2}\left|V_{12}\right|^{2} \mathcal{A}(T) F^{(1)} F^{(2)},
$$

where the integration is taken over transferred momentum and energy in the intershell interaction, and the summation includes all involved bands and other quantum numbers required to specify the states. $\mathcal{A}(T)$ is a thermal factor, $V_{12}$ is the screened intershell Coulomb interaction, and the $F$-functions for the two subsystems account for the available phase-space for electronic scattering. Of crucial importance is the factor SR accounting for the selection rules (or rather suppression rules) stemming from the intershell Coulomb 
matrix element between the Bloch states. (In the final formula some $\mathrm{SR}$ is incorporated into the $F$ functions.) As known from experimental ${ }^{15}$ and theoretical ${ }^{68-70}$ studies, backscattering between the linear bands in metallic tubes by impurities with slowly varying potentials are strongly suppressed leading to very long mean free paths. The selection rules for intershell Coulomb interaction lead to a similar suppression, which depends strongly on the inner and outer tubes' chirality. A detailed analysis of these effects is one of the central tasks of the present article. The structure of Eq. (1) is much richer than its counterparts' for coupled quantum wells due to the rather complicated band structure combinations of the various MWCNT's.

\section{CARBON NANOTUBE BAND STRUCTURE}

In Appendix A, we give a detailed account for the band structure of a SWCNT with chirality $(n, m)$, since it turns out to be of crucial importance to the intershell Coulomb matrix element and thereby also for the drag. Here we only outline the important points of the band structure used later. The carbon nanotube band structure can be found by applying periodic boundary conditions to the band structure of a single graphite layer (graphene). Graphene has two atoms in the primitive unit cell, so the tight-binding state (or Wannier decomposition) have two components with weights $\alpha$ and $\beta$ [see Eq. (A5)]. When applying the periodic boundary condition the wave vector component around the tube $k_{c}$ becomes quantized into discrete values, $k_{c}=(2 \pi /|\mathbf{C}|) n_{c}$. However, it is important to realize that $n_{c}$ is not the crystal angular momentum $\mathfrak{m}$ stemming from the rotation symmetry, but only related to it by $n_{c}=\mathfrak{m}(\bmod \mathfrak{n})$. [Here $\mathbf{C}$ is the chiral vector and $\mathfrak{n}$ is the greatest common divisor of $(n, m), \mathfrak{n}=\operatorname{gcd}(n, m)$.] This is due to the nonprimitive (large) nanotube unit cell, when using translational symmetry instead of helical symmetry. ${ }^{71,72}$

Linearizing the tight-binding band structure around the Fermi level $\varepsilon_{\mathrm{F}}=0$ the states and bands for metallic tubes near $\varepsilon_{\mathrm{F}}$ become

$$
\begin{gathered}
\varepsilon_{\mathfrak{K}_{T}}^{\xi}=\xi v_{0} \mathfrak{K}_{T}, \\
\left(\begin{array}{l}
\alpha \\
\beta
\end{array}\right)_{\xi, \varsigma}=\frac{1}{\sqrt{2}}\left(\begin{array}{c}
-\xi \frac{i(m-n)-\varsigma \sqrt{3}(n+m)}{2 \sqrt{n^{2}+m^{2}+m n}} \\
1
\end{array}\right),
\end{gathered}
$$

where $\mathfrak{K}_{T}$ is the wave vector along the tube measured from the point, where the band crosses $\varepsilon_{\mathrm{F}}=0\left(\mathfrak{K}_{T}=k-k_{\varepsilon_{\mathrm{F}}=0}\right), \xi$ $= \pm 1$ is the sign of the velocity in the band and $s= \pm 1$ describes which $\mathbf{K}_{\mathrm{s}}$ point of graphene the linear band originate from. Here $v_{0}=\left(\sqrt{3} \gamma_{0} a / 2 \hbar\right)$ with $\gamma_{0} \simeq 3 \mathrm{eV}$ and $a \equiv \sqrt{3} a_{\mathrm{c}-\mathrm{c}}$ $\left(a_{\mathrm{c}-\mathrm{c}}=0.142 \mathrm{~nm}\right)$. The metallic states can thus described by $(k, \xi, \varsigma)$. Using this, we can classify all metallic tubes into two categories: zigzaglike (ZL) and armchairlike ( $A L)$ tubes, with the following bands near the Fermi level (shown on Fig. 2):

$$
\text { zigzaglike: } \varepsilon_{k \mathfrak{m}}^{\xi}=\xi \hbar v_{0} k, \quad \mathfrak{m} \in\left\{\mathfrak{m}_{a}, \mathfrak{m}_{b}\right\}
$$

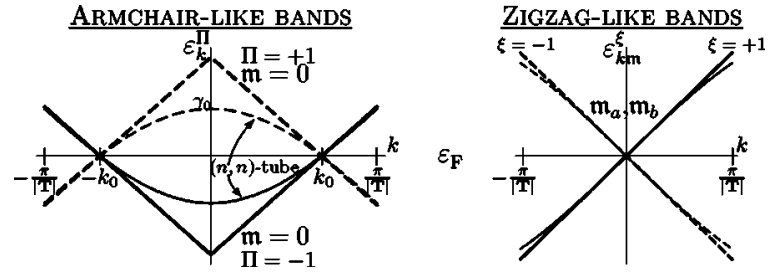

FIG. 2. The two categories of metallic nanotubes: armchairlike (AL, left) and zigzaglike (ZL, right). The AL bands near $\varepsilon_{\mathrm{F}}=0$ have zero crystal angular momentum $\mathfrak{m}=0$ and $\Pi= \pm 1$, where $k_{0}$ $\equiv 2 \pi / 3|\mathbf{T}|$. The ZL tubes have doubly degenerate bands crossing $\varepsilon_{\mathrm{F}}=0$, i.e., for each $\xi= \pm 1$ we have either $\mathfrak{m}_{a}=(2 n+m) / 3(\bmod \mathfrak{n})$ or $\mathfrak{m}_{b}=(2 m+n) / 3(\bmod \mathfrak{n})$, where $\mathfrak{m}_{a} \neq \mathfrak{m}_{b}[\mathfrak{n}=\operatorname{gcd}(n, m)]$. The thin lines are the tight-binding bands near $\varepsilon_{\mathrm{F}}=0$ for a $(n, n)$ tube (with $|\mathbf{T}|=a)$ and $\mathrm{a}(3 m, 0)$ tube.

$$
\text { armchairlike: } \varepsilon_{k}^{\Pi}=-\Pi \hbar v_{0}\left(|k|-k_{0}\right), \quad \mathfrak{m}=0 .
$$

Here $k \in]-\pi /|\mathbf{T}|, \pi /|\mathbf{T}|]$ is the wave vector along the tube, $k_{0}=2 \pi / 3|\mathbf{T}|$ and $\mathbf{T}$ is the translational vector generating the translational symmetry. Note that two different tubes can have different $|\mathbf{T}|$ even though they belong to the same category. $\Pi= \pm 1$ originates from $\xi$, but does not give the sign of the velocity, and for a $(n, n)$ tube $\Pi$ is the parity in the cylindrical angle..$^{73,74}$ The linearity of the bands near the Fermi level is, of course, well known, but it is important to recognize the entirely different angular momentum quantum numbers $\mathfrak{m}$ that characterize the $\mathrm{AL}$ and $\mathrm{ZL}$ bands crossing the Fermi level. Specifically, for AL tubes it always holds that $\mathfrak{m}=0$, while for the $\mathrm{ZL}$ tube one has

$$
\mathfrak{m}_{a}=\frac{2 n+m}{3}(\bmod \mathfrak{n}) \text { or } \mathfrak{m}_{b}=\frac{2 m+n}{3}(\bmod \mathfrak{n}) .
$$

Note that $\mathfrak{m}_{a} \neq \mathfrak{m}_{b}$ and $\mathfrak{m}_{a}, \mathfrak{m}_{b}$ are never zero. There is a one-to-one correspondence between $s= \pm 1$ and the crystal angular momentum of the linear bands. We note that the most commonly studied metallic zigzag and armchair tubes, with indices $(3 n, 0)$ and $(n, n)$, are of course special cases of $\mathrm{ZL}$ and AL tubes, respectively.

\section{INTERSHELL COULOMB INTERACTION}

We next consider the Coulomb interaction between Bloch states $\left|k n_{c}\right\rangle$ for electrons in different shells in a MWCNT. Before calculating the Coulomb matrix element involving products $^{75}$ of Bloch states it is useful to consider the less complicated problem of the impurity matrix element $\left\langle k^{\prime} n_{c}^{\prime}|V(\mathbf{r})| k n_{c}\right\rangle$. The essential assumption that we use in calculating both the impurity and Coulomb matrix element is that the potential is slowly varying on the scale of the interatomic distance $a_{\mathrm{c}-\mathrm{c}}$. In the case of impurity scattering this is a fair assumption for an impurity held on the tube by van der Waals forces as is often the case. ${ }^{76,77}$ For Coulomb interaction between different shells it is also a good assumption, since the electrons do not get close enough to experience the $1 / r$ singularity.

The impurity matrix element $\left\langle\mathbf{k}^{\prime}|V(\mathbf{r})| \mathbf{k}\right\rangle$ between the two component Bloch states $\psi_{\mathbf{k}}(\mathbf{r})$, Eq. (A5) (before applying periodic boundary conditions), is 


$$
\begin{aligned}
\left\langle\mathbf{k}^{\prime}|V(\mathbf{r})| \mathbf{k}\right\rangle & \\
= & \frac{1}{N} \sum_{\mathbf{R}, \mathbf{R}^{\prime}} e^{-i \mathbf{k}^{\prime} \cdot \mathbf{R}^{\prime}+i \mathbf{k} \cdot \mathbf{R}} \\
& \times\left[\alpha_{\mathbf{k}^{\prime}}^{*} \alpha_{\mathbf{k}} \int \mathrm{d} \mathbf{r} \Psi^{*}\left(\mathbf{r}-\mathbf{R}^{\prime}\right) V(\mathbf{r}) \Psi(\mathbf{r}-\mathbf{R})\right. \\
& +\alpha_{\mathbf{k}^{\prime}}^{*} \beta_{\mathbf{k}} \int \mathrm{d} \mathbf{r} \Psi^{*}\left(\mathbf{r}-\mathbf{R}^{\prime}\right) V(\mathbf{r}) \Psi(\mathbf{r}-\mathbf{R}-\mathbf{d}) \\
& +\beta_{\mathbf{k}^{\prime}}^{*} \alpha_{\mathbf{k}} \int \mathrm{d} \mathbf{r} \Psi^{*}\left(\mathbf{r}-\mathbf{R}^{\prime}-\mathbf{d}\right) V(\mathbf{r}) \Psi(\mathbf{r}-\mathbf{R}) \\
& \left.+\beta_{\mathbf{k}^{\prime}}^{*} \beta_{\mathbf{k}} \int \mathrm{d} \mathbf{r} \Psi^{*}\left(\mathbf{r}-\mathbf{R}^{\prime}-\mathbf{d}\right) V(\mathbf{r}) \Psi(\mathbf{r}-\mathbf{R}-\mathbf{d})\right] .
\end{aligned}
$$

By using the assumption of slow variation of $V(\mathbf{r})$ we can take the potential outside the integrals. The first and last terms in the square brackets become $\delta_{\mathbf{R}^{\prime}, \mathbf{R}} V(\mathbf{R})\left(\alpha_{\mathbf{k}^{\prime}}^{*} \alpha_{\mathbf{k}}\right.$ $\left.+\beta_{\mathbf{k}^{\prime}}^{*}, \beta_{\mathbf{k}}\right)$ and the second and third terms are found (including a sum) by summing over the nearest neighbors to be ${ } s_{0}\left[\alpha_{\mathbf{k}^{\prime}}^{*} \beta_{\mathbf{k}} \Upsilon\left(\mathbf{k}^{\prime}\right)+\alpha_{\mathbf{k}} \beta_{\mathbf{k}^{\prime}}^{*} \Upsilon^{*}(\mathbf{k})\right]$. Equation (A7) defines $\Upsilon(\mathbf{k})$ and $s_{0} \sim 0.1$ is the overlap between neighboring orbitals (see Appendix A). Introducing the Fourier transform of the potential $V(\mathbf{k})$ and the reciprocal lattice vector $\mathbf{G}$ we find

$$
\left\langle\mathbf{k}^{\prime}|V(\mathbf{r})| \mathbf{k}\right\rangle=g\left(\mathbf{k}, \mathbf{k}^{\prime}\right) \frac{1}{\mathcal{A}} \sum_{\mathbf{G}} V\left(\mathbf{k}^{\prime}-\mathbf{k}+\mathbf{G}\right),
$$

where $\mathcal{A}$ is the surface area and the $g$ factor is

$$
g\left(\mathbf{k}, \mathbf{k}^{\prime}\right) \equiv \alpha_{\mathbf{k}} \alpha_{\mathbf{k}^{\prime}}^{*}+\beta_{\mathbf{k}} \beta_{\mathbf{k}^{\prime}}^{*}+s_{0}\left[\alpha_{\mathbf{k}^{\prime}}^{*} \beta_{\mathbf{k}} \Upsilon\left(\mathbf{k}^{\prime}\right)+\alpha_{\mathbf{k}} \beta_{\mathbf{k}^{\prime}}^{*} \Upsilon^{*}(\mathbf{k})\right]
$$

i.e., the matrix element is essentially the plane wave result times a band structure factor, which we will refer to as the $g$ factor.

To obtain the matrix element for the screened Coulomb interaction $V\left(\mathbf{r}_{1}, \mathbf{r}_{2}\right)$ (suppressing the frequency argument $\omega$ in the notation) we note that $\left\langle\mathbf{k}_{1}^{\prime} \mathbf{k}_{2}^{\prime}\left|V\left(\mathbf{r}_{1}, \mathbf{r}_{2}\right)\right| \mathbf{k}_{1} \mathbf{k}_{2}\right\rangle$ $=\left\langle\mathbf{k}_{2}^{\prime}\left|\left\langle\mathbf{k}_{1}^{\prime}\left|V\left(\mathbf{r}_{1}, \mathbf{r}_{2}\right)\right| \mathbf{k}_{1}\right\rangle\right| \mathbf{k}_{2}\right\rangle$, where $i=1,2$ labels the outer $/$ inner tube, respectively. Therefore we can use the impurity potential result, Eq. (8), to obtain

$$
\begin{aligned}
\left\langle\mathbf{k}_{1}^{\prime} \mathbf{k}_{2}^{\prime}\right| & V\left(\mathbf{r}_{1}, \mathbf{r}_{2}\right)\left|\mathbf{k}_{1} \mathbf{k}_{2}\right\rangle \\
= & g_{1}\left(\mathbf{k}_{1}, \mathbf{k}_{1}^{\prime}\right) g_{2}\left(\mathbf{k}_{2}, \mathbf{k}_{2}^{\prime}\right) \\
& \times \frac{1}{\mathcal{A}_{1} \mathcal{A}_{2}} \sum_{\mathbf{G}_{1}, \mathbf{G}_{2}} V\left(\mathbf{k}_{1}^{\prime}-\mathbf{k}_{1}+\mathbf{G}_{1}, \mathbf{k}_{2}^{\prime}-\mathbf{k}_{2}+\mathbf{G}_{2}\right),
\end{aligned}
$$

where we have a $g$ factor for each system and the screened potential is Fourier transformed separately in both $\mathbf{r}_{1}$ and $\mathbf{r}_{2}$.

For a $\left(n_{2}, m_{2}\right)$ tube inside a $\left(n_{1}, m_{1}\right)$ tube the screened Coulomb matrix element is found using cylindrical coordinates $\mathbf{r}=(r, \theta, z)$ to be

$$
\begin{aligned}
\left\langle k_{1}^{\prime} n_{c_{1}}^{\prime} k_{2}^{\prime} n_{c_{2}}^{\prime}\left|V\left(\mathbf{r}_{1}, \mathbf{r}_{2}\right)\right| k_{1} n_{c_{1}} k_{2} n_{c_{2}}\right\rangle= & \frac{1}{(2 \pi L)^{2}} g_{1}\left(k_{1} n_{c_{1}}, k_{1}^{\prime} n_{c_{1}}^{\prime}\right) g_{2}\left(k_{2} n_{c_{2}}, k_{2}^{\prime} n_{c_{2}}^{\prime}\right) \sum_{G_{1}, G_{2}} \sum_{u_{1}, u_{2} \in \mathbb{Z}} \\
& V\left(k_{1}^{\prime}-k_{1}+G_{1}, \mathfrak{m}_{1}^{\prime}-\mathfrak{m}_{1}+\mathfrak{n}_{1} u_{1}, k_{2}^{\prime}-k_{2}+G_{2}, \mathfrak{m}_{2}^{\prime}-\mathfrak{m}_{2}+\mathfrak{n}_{2} u_{2}, r_{1}, r_{2}\right),
\end{aligned}
$$

where $L$ is the length of tubes, $\mathfrak{n}_{i}=\operatorname{gcd}\left(n_{i}, m_{i}\right), G_{i}$ $=\left(2 \pi /\left|\mathbf{T}_{i}\right|\right)_{s}(s \in \mathbb{Z})$, and $r_{i}$ is the radius ${ }^{78}$ of tube $i$.

We will also need the unscreened Coulomb matrix element $V^{0}$, which is a function of the interparticle distance $\left|\mathbf{r}_{1}-\mathbf{r}_{2}\right|$, i.e., a function of $z_{1}-z_{2}, \theta_{1}-\theta_{2}, r_{1}$, and $r_{2}$, so we Fourier transform in the differences $z_{1}-z_{2}$ and $\theta_{1}-\theta_{2}$. Therefore this matrix element is

$$
\begin{aligned}
& \left\langle k_{1}^{\prime} n_{c_{1}}^{\prime} k_{2}^{\prime} n_{c_{2}}^{\prime}\left|V^{0}\left(\left|\mathbf{r}_{1}-\mathbf{r}_{2}\right|\right)\right| k_{1} n_{c_{1}} k_{2} n_{c_{2}}\right\rangle \\
& =\frac{1}{2 \pi L} g_{1}\left(k_{1} n_{c_{1}}, k_{1}^{\prime} n_{c_{1}}^{\prime}\right) g_{2}\left(k_{2} n_{c_{2}}, k_{2}^{\prime} n_{c_{2}}^{\prime}\right) \\
& \quad \times \sum_{G_{1}, G_{2}} \sum_{u_{1}, u_{2} \in Z} V^{0}\left(k_{1}^{\prime}-k_{1}+G_{1}, \mathfrak{m}_{1}^{\prime}-\mathfrak{m}_{1}+\mathfrak{n}_{1} u_{1}, r_{1}, r_{2}\right) \\
& \quad \times \delta_{k_{1}+k_{2}, k_{1}^{\prime}+k_{2}^{\prime}+G_{1}+G_{2}} \delta_{\mathfrak{m}_{1}^{\prime}+\mathfrak{m}_{2}^{\prime}+\mathfrak{n}_{1} u_{1}, \mathfrak{m}_{1}+\mathfrak{m}_{2}+\mathfrak{n}_{2} u_{2}} .
\end{aligned}
$$

Note that the \pm in the states Eq. (A11) [the $\xi$ index for metallic states Eqs. (2) and (3)] is suppressed in the notation and that this index only appears in the g factors in both Eqs. (11) and (12). Here we have used the crystal angular momentum difference in the Fourier transforms instead of the $n_{c}$ difference, since this is the physical (crystal) angular momentum being transferred. ${ }^{79}$ Note that we have included umklapp scattering and that the unscreened interaction Eq. (12) has crystal (angular) momentum conservation. Similar matrix elements were considered by Uryu. ${ }^{44}$

\section{A. The $g$ factor and backscattering in metallic tubes}

We now consider the $g$ factors and show that they contain essential information about the electronic scattering. The $g$ factor for any $(n, m)$ metallic tube for the scattering process $(k, \xi, \varsigma) \rightarrow\left(k^{\prime}, \xi^{\prime}, \varsigma^{\prime}\right)$ between the metallic states, Eq. (3), is found by inserting Eq. (A10) (with $\mathfrak{K}=\mathfrak{K}_{T} \mathbf{T} /|\mathbf{T}|$ ) and Eq. (3) into Eq. (9): 


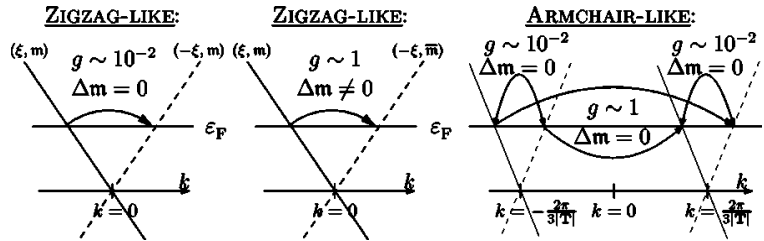

FIG. 3. The possible backscattering processes in any metallic tube with a slightly raised Fermi level $\varepsilon_{\mathrm{F}}$. Left: Backscattering in a zigzaglike tube without crystal angular momentum change $\Delta \mathfrak{m}=0$ (i.e., $\varsigma=\varsigma^{\prime}$ ) and a small wave vector $\left|k^{\prime}-k\right| \sim 2 \varepsilon_{\mathrm{F}} / \hbar v_{0}$ change, which is suppressed by $g \lesssim 10^{-2}$ from Eq. (15). Center: Backscattering in a zigzaglike tube with crystal angular momentum change, which have $g \sim 1$ from Eq. (16). Here $\overline{\mathfrak{m}}$ denotes the opposite of $\mathfrak{m}$ in the set $\left\{\mathfrak{m}_{a}, \mathfrak{m}_{b}\right\}$. Right: Two types of backscattering in armchairlike tubes: (i) A large wave vector transfer (for $s=-s^{\prime}$ ) between states with the same crystal angular momentum $(\mathfrak{m}=0)$ and $g \sim 1$ [Eq. (16)] and (ii) a small wave vector transfer $q \simeq 2 \varepsilon_{\mathrm{F}} / \hbar v_{0}$ suppressed by $g \leqq 10^{-2}$. Note that the distance between the points $\pm 2 \pi / 3|\mathbf{T}|$ are not to scale (i.e., $2 \varepsilon_{\mathrm{F}} / \hbar v_{0} \ll 4 \pi / 3|\mathbf{T}|$ ) and that the armchairlike bands are connected as in Fig. 2.

$$
g\left(k, \mathrm{~s}, \xi ; k^{\prime}, \mathrm{s}^{\prime}, \xi^{\prime}\right)=\frac{1}{2}\left(\xi \xi^{\prime} f_{n, m, \mathrm{~s}, \mathrm{~s}^{\prime}}+1\right)-s_{0} \frac{\sqrt{3} a\left(\xi^{\prime} \mathfrak{K}_{T}^{\prime}+\xi \mathfrak{K}_{T}\right)}{4}
$$

where we introduced

$$
\begin{aligned}
f_{n, m, \mathrm{~s}, \mathrm{~s}^{\prime}}= & \delta_{\mathrm{s}, \mathrm{s}^{\prime}}-\frac{n^{2}+m^{2}+4 m n}{2\left(n^{2}+m^{2}+m n\right)}\left(1-\delta_{\mathrm{s}, \mathrm{s}^{\prime}}\right) \\
& +i \frac{\sqrt{3} \mathrm{~s}\left(m^{2}-n^{2}\right)}{2\left(n^{2}+m^{2}+m n\right)}\left(1-\delta_{\mathrm{s}, \mathrm{s}^{\prime}}\right) .
\end{aligned}
$$

The $g$ factor in Eq. (13) has two terms: The first parentheses is the important wave vector independent scalar product of $\left(\begin{array}{l}\alpha \\ \beta\end{array}\right)$ from Eq. (3) and the second term is a wave vector dependent correction term (of first order in $s_{0} \sim 0.1$ ).

As we shall show in Sec. IV, only backscattering contributes to the Coulomb drag in metallic tubes and we therefore need to consider all possible backscattering processes $\left(\xi=-\xi^{\prime}\right)$ in any metallic tube. Due to the double degeneracy of the zigzaglike bands Eq. (4) at the Fermi level, we must consider backscattering both with and without crystal momentum exchange (Fig. 3, center and left panels, respectively).

If $s=s^{\prime}$, then $\Delta \mathfrak{m} \equiv \mathfrak{m}^{\prime}-\mathfrak{m}=0$ and from Eq. (13) we have

$$
\left|g\left(k, \varsigma, \xi ; k^{\prime}, \varsigma,-\xi\right)\right|=s_{0} \frac{\sqrt{3} a\left|k^{\prime}-k\right|}{4}
$$

which is of order $10^{-2}$ or less for scattering around the Fermi level, i.e. for $\left|k^{\prime}-k\right| \simeq 2\left|\varepsilon_{\mathrm{F}}\right| / \hbar v_{0}$ the $g$ factor is $|g|$ $=s_{0}\left|\varepsilon_{\mathrm{F}}\right| / \gamma_{0} \lesssim 10^{-2}$ for $\left|\varepsilon_{\mathrm{F}}\right| \lesssim 0.3 \mathrm{eV}$. If $\varsigma=-\varsigma^{\prime}$ then $|\Delta \mathfrak{m}|$ $=\left|\mathfrak{m}_{a}-\mathfrak{m}_{b}\right| \neq 0$ and for backscattering around the Fermi level the $g$ factor squared is ${ }^{80}$

$$
\left|g_{\varepsilon \sim \varepsilon_{\mathrm{F}}}\right|^{2} \simeq \frac{1}{4}\left(1+\frac{n^{2}+m^{2}+4 m n}{2\left(n^{2}+m^{2}+m n\right)}\right)^{2}+\frac{3}{16}\left(\frac{m^{2}-n^{2}}{n^{2}+m^{2}+m n}\right)^{2},
$$

which is $\frac{3}{4}$ for $(n, 0), 1$ for $(n, n)$ and in between for all other tubes. So in a zigzaglike tube we have two kinds of backscattering with small crystal wave vector exchange $q$ $\sim 2 \varepsilon_{\mathrm{F}} / \hbar v_{0}$ [and thereby large $\left.V(q, \Delta \mathfrak{m})\right]$ : Either $\Delta \mathfrak{m}=0$ and $|g| \lesssim 10^{-2}$ or $\Delta \mathfrak{m} \neq 0$ and $|g| \sim 1$. Note that the larger the $\Delta \mathfrak{m}$ the smaller $V(q, \Delta \mathfrak{m})$. Even though $V(q, \Delta \mathfrak{m})$ is large the small $g$ factor suppresses the $\Delta \mathfrak{m}=0$ backscattering.

Consider now armchairlike tubes where the bands crossing $\varepsilon_{\mathrm{F}}=0$ all have $\mathfrak{m}=0$, so the small crystal wave vector transfer around $\pm 2 \pi / 3|\mathbf{T}|$ have $s=s^{\prime}$ and therefore the $g$ factor is the same as in Eq. (15), i.e., $|g| \lesssim 10^{-2}$ suppresses this kind of backscattering [Fig. 3 (right)]. If we on the other hand have a large crystal wave vector transfer backscattering [Fig. 3 (right)], then $s=-s^{\prime}$ and the $g$ factor of order 1 from Eq. (16) is used. So the large crystal wave vector backscattering is most important, since the Fourier transform does not grow enough to compensate for the small $g$ factor.

Ando et al. ${ }^{68,69}$ have used the $\mathbf{k} \cdot \mathbf{p}$ approximation to consider backscattering (from impurities) in metallic tubes and found a result similar to Eq. (9), but without the $s_{0}$ term. The small wave vector transfer backscattering was found to be small in these papers. Klesse ${ }^{70}$ has found similar results for scattering in nanotubes; see also Ref. 15 for some experimental evidence of lack of backscattering in metallic tubes compared to semiconducting ones.

\section{B. Screening effects using the random phase approximation}

In Appendix B, we derive the screened Coulomb interaction in the random phase approximation (RPA) including the carbon nanotube band structure with the result

$$
\begin{aligned}
\left\langle k_{1}^{\prime} \mathfrak{m}_{1}^{\prime} \xi_{1}^{\prime}, k_{2}^{\prime} \mathfrak{m}_{2}^{\prime} \xi_{2}^{\prime}\left|V\left(\mathbf{r}_{1}, \mathbf{r}_{2}, \omega\right)\right| k_{1} \mathfrak{m}_{1} \xi_{1}, k_{2} \mathfrak{m}_{2} \xi_{2}\right\rangle & =\frac{1}{2 \pi L} g_{1}\left(k_{1} \varsigma_{1} \xi_{1}, k_{1}^{\prime} \boldsymbol{s}_{1}^{\prime} \xi_{1}^{\prime}\right) g_{2}\left(k_{2} \varsigma_{2} \xi_{2}, k_{2}^{\prime} \varsigma_{2}^{\prime} \xi_{2}^{\prime}\right) \\
& \times \sum_{G_{1}, G_{2}} \sum_{u_{1}, u_{2} \in \mathbb{Z}} \frac{V^{0}\left(k_{1}^{\prime}-k_{1}+G_{1}, \mathfrak{m}_{1}^{\prime}-\mathfrak{m}_{1}+\mathfrak{n}_{1} u_{1}, r_{1}, r_{2}\right)}{\epsilon_{12}\left(k_{1}^{\prime}-k_{1}, \mathfrak{m}_{1}^{\prime}-\mathfrak{m}_{1}, \omega\right)} \\
& \times \delta_{k_{1}+k_{2}, k_{1}^{\prime}+k_{2}^{\prime}+G_{1}+G_{2}} \delta_{\mathfrak{m}_{1}^{\prime}+\mathfrak{m}_{2}^{\prime}+\mathfrak{n}_{1} u_{1}, \mathfrak{m}_{1}+\mathfrak{m}_{2}+\mathfrak{n}_{2} u_{2},}
\end{aligned}
$$

where $\epsilon_{12}(q, \Delta \mathfrak{m}, \omega)$ is the dielectric function disregarding the umklapp processes [see Eq. (B10)]. Note that the effective noninteracting polarization $\chi_{\text {eff }, i}^{0}(q, \Delta \mathfrak{m}, \omega)$, Eq. (B8), entering the dielectric function contains the $g$ factors. For armchairlike tubes $\chi_{\text {eff }, i}^{0}(q, \Delta \mathfrak{m}, \omega)$ is given explicitly in Eqs. (B12) and (B13). The bare Coulomb interaction for a cylindrical geometry is

$$
V^{0}\left(q, \Delta \mathfrak{m}, r_{i}, r_{j}\right)=\frac{e^{2}}{\epsilon_{0}} \mathrm{I}_{\Delta \mathfrak{m}}\left(q r_{i}\right) \mathrm{K}_{\Delta \mathfrak{m}}\left(q r_{j}\right), \quad r_{i} \leqslant r_{j},
$$

where $\mathrm{I}_{\Delta \mathfrak{m}}(x)\left[\mathrm{K}_{\Delta \mathfrak{m}}(x)\right]$ is the modified Bessels functions of the first [second] kind of order $\Delta \mathfrak{m}$ and $\epsilon_{0}$ is the vacuum permittivity. 


\section{THE TRANSRESISTANCE MODEL}

The transresistance $R_{21}$ is now found for diffusive nanotubes using two coupled Boltzmann equations (i.e., Fermi liquid theory) in linear response to the applied electric field $E_{1}$ and for weak coupling between the tubes. The derivation is a generalization of Refs. 55 and 81-83 (used to study bilayer systems) to the case of several general bands. We only sketch the derivation and the details can be found in Chap. 3 of Ref. 80. In order to simplify the notation we use $\nu$ as a collection of band indices for the tube. A similar formula of $R_{21}$ can also be found using the Kubo formula and doing perturbation theory to second order in the intertube interaction (the first order dc contribution is zero). ${ }^{84}$

The coupled linearized Boltzmann equations for the nonequilibrium distribution functions $f_{i}\left(k_{i}, \nu_{i}\right) \quad(i=1,2$; see Fig. 1) are

$$
\begin{aligned}
\frac{e_{1} E_{1}}{\hbar} \frac{\partial f^{0}\left(\varepsilon_{k_{1} \nu_{1}}\right)}{\partial k_{1}}= & -\frac{f_{1}\left(k_{1}, \nu_{1}\right)-f^{0}\left(\varepsilon_{k_{1} \nu_{1}}\right)}{\tau_{1}}, \\
\frac{e_{2} E_{2}}{\hbar} \frac{\partial f^{0}\left(\varepsilon_{k_{2} \nu_{2}}\right)}{\partial k_{2}}= & -\frac{f_{2}\left(k_{2}, \nu_{2}\right)-f^{0}\left(\varepsilon_{k_{2} \nu_{2}}\right)}{\tau_{2}} \\
& +S\left[f_{1}, f_{2}=f^{0}\right]\left(k_{2}, \nu_{2}\right),
\end{aligned}
$$

where a simple relaxation time approximation is used for the impurity scattering, ${ }^{85} e_{i}$ is the carrier charge in subsystem $i$ and $S\left[f_{1}, f_{2}=f^{0}\right]$ is the linearized collision integral coupling the two subsystems/tubes. The assumption of weak intertube interaction and small external electric field $E_{1}$ were used to linearize the equations and to only include the lowest-order terms and therefore not have a collision integral on Eq. (19). The linearized collision integral is (using the $H$ theorem $^{85}$ ):

$$
\begin{aligned}
S\left[f_{1}, f_{2}\right. & \left.=f^{0}\right]\left(k_{2}, \nu_{2}\right) \\
= & -\sum_{\sigma_{1} \sigma_{1}^{\prime} \sigma_{2}^{\prime}} \sum_{\nu_{1} \nu_{1}^{\prime} \nu_{2}^{\prime} k_{1}, k_{1}^{\prime}, k_{2}^{\prime} \in \mathrm{FBZ}} w\left(1^{\prime} 2^{\prime} ; 12\right) f^{0}\left(\varepsilon_{k_{1} \nu_{1}}\right) f^{0}\left(\varepsilon_{k_{2} \nu_{2}}\right) \\
& \times\left[1-f^{0}\left(\varepsilon_{k_{1}^{\prime} \nu_{1}^{\prime}}\right)\right]\left[1-f^{0}\left(\varepsilon_{k_{2}^{\prime} \nu_{2}^{\prime}}\right)\right]\left[\psi_{1}\left(k_{1}, \nu_{1}\right)-\psi_{1}\left(k_{1}^{\prime}, \nu_{1}^{\prime}\right)\right],
\end{aligned}
$$

where the deviation from equilibrium $\psi_{i}(k, \nu)$ was defined though $f_{i}(k, \nu)-f^{0}\left(\varepsilon_{k \nu}\right) \equiv f^{0}\left(\varepsilon_{k \nu}\right)\left[1-f^{0}\left(\varepsilon_{k \nu}\right)\right] \psi_{i}(k, \nu) \quad$ and $w\left(1^{\prime} 2^{\prime} ; 12\right)$ is the transition rate for electron-electron scattering between the tubes found from the Fermi golden rule

$$
\begin{aligned}
w\left(1^{\prime} 2^{\prime} ; 12\right)= & \frac{2 \pi}{\hbar}\left|\left\langle k_{1}^{\prime} \nu_{1}^{\prime} k_{2}^{\prime} \nu_{2}^{\prime}\left|V_{12}\left(\left|\mathbf{r}_{1}-\mathbf{r}_{2}\right|\right)\right| k_{1} \nu_{1} k_{2} \nu_{2}\right\rangle\right|^{2} \\
& \times \delta\left(\varepsilon_{k_{1} \nu_{1}}+\varepsilon_{k_{2} \nu_{2}}-\varepsilon_{k_{1}^{\prime} \nu_{1}^{\prime}}-\varepsilon_{k_{2}^{\prime} \nu_{2}^{\prime}}\right)
\end{aligned}
$$

using the matrix element in Eq. (17). To derive the transresistance $R_{21}=V_{2} / I_{1}$, we use the coupled Boltzmann equations (19) and (20) with (21) and that $I_{2}=0$, since a voltmeter is placed on subsystem $2 .{ }^{81}$ After some algebra ${ }^{80}$ we get

$$
\begin{aligned}
R_{21}= & \frac{\hbar^{2}}{\pi e_{1} e_{2} n_{1} n_{2} k_{\mathrm{B}} T} \frac{L}{(2 \pi)^{2} r_{1} r_{2}} \sum_{G_{1} G_{2}} \delta_{G_{1}, G_{2}} \\
& \times \frac{1}{(2 \pi)^{2}} \sum_{\nu_{1} \nu_{1}^{\prime} \nu_{2} \nu_{2}^{\prime}}\left|\mathcal{J}\left(\nu_{1} \nu_{1}^{\prime}, \nu_{2} \nu_{2}^{\prime}\right)\right|^{2} \\
& \times \int_{0}^{\infty} \frac{d q}{2 \pi} \int_{0}^{\infty} d \omega \frac{V_{12}\left(q, \nu_{1}, \nu_{1}^{\prime}, \omega\right) V_{12}^{*}\left(q+G_{1}, \nu_{1}, \nu_{1}^{\prime}, \omega\right)}{\sinh ^{2}\left(\hbar \omega / 2 k_{\mathrm{B}} T\right)} \\
& \times F_{\nu_{1} \nu_{1}^{\prime}}^{(1)}(q, \omega) F_{\nu_{2} \nu_{2}^{\prime}}^{(2)}(q, \omega),
\end{aligned}
$$

where $n_{i}$ is the carrier density,

$$
V_{12}\left(q, \nu_{1}, \nu_{1}^{\prime}, \omega\right)=\frac{V^{0}\left(q, \Delta \mathfrak{m}, r_{1}, r_{2}\right)}{\epsilon_{12}(q, \Delta \mathfrak{m}, \omega)}
$$

from Eq. (17), $\mathcal{J}\left(\nu_{1} \nu_{1}^{\prime}, \nu_{2} \nu_{2}^{\prime}\right)$ are the selection rules for the band indices such as crystal angular momentum and/or parity (for armchair tubes) conservation and $F_{\nu_{i} \nu_{i}^{\prime}}^{(i)}(q, \omega)$ is the available $(q, \omega)$-phase space for scattering in the $i$ th tube given by

$$
\begin{aligned}
F_{\nu_{i} \nu_{i}^{\prime}}^{(i)}(q, \omega)= & -\frac{e_{i} \tau_{i}}{\hbar^{2} \mu_{\operatorname{Tr}}^{(i)} \sum_{s}} \operatorname{sgn}\left(v_{k_{s} \nu_{i}}-v_{k_{s}+q \nu_{i}^{\prime}}\right) \\
& \times\left[f^{0}\left(\varepsilon_{k_{s} \nu_{i}}\right)-f^{0}\left(\varepsilon_{k_{s}+q \nu_{i}^{\prime}}\right)\right]\left|g_{i}\left(k_{s} \nu_{i}, k_{s}+q \nu_{i}^{\prime}\right)\right|^{2},
\end{aligned}
$$

where the $k_{s}$ are the solutions to $\varepsilon_{k \nu_{i}}-\varepsilon_{k+q \nu_{i}^{\prime}}-\hbar \omega=0$ in the first Brillouin zone (FBZ) of subsystem $i$,

$$
v_{k \nu}=\frac{1}{\hbar} \frac{\partial \varepsilon_{k \nu}}{\partial k}
$$

is the velocity, $\operatorname{sgn}(x)$ gives the sign of $x$ [if $x=0$ then $\operatorname{sgn}(x)=0]$, and $\mu_{\operatorname{Tr}}^{(i)}$ is the transport mobility, which is a single subsystem property. Note that the $F$ function is periodic and odd in $q$.

Having stated this formula a few comments and interpretations are in order. First, we note that only backscattering processes contribute to the drag between metallic tubes in the linearized band models, Eqs. (4) and (5), since we only have two velocities $\pm v_{0}= \pm \sqrt{3} \gamma_{0} a / 2 \hbar$ in the metallic bands, and therefore the signum-function of the velocity difference before and after the scattering event in the $F$ function, Eq. (23), makes only backscattering (i.e., $v_{k_{s} \nu_{i}}=-v_{k_{s}+q v_{i}^{\prime}}$ ) contribute to the $F$ function. In Sec. III A we therefore analyzed the $g$ factors for all possible backscattering processes in metallic tubes. The interaction and $\sinh ^{-2}\left(\hbar \omega / 2 k_{\mathrm{B}} T\right)$ are decreasing functions of $q$ and $\omega$, respectively, so the importance of the phase space (i.e., the $F$ functions) in the integral decreases from the origin. It is worth noting that the forward scattering contribution, which for quadratic dispersion relation dominates at higher temperatures ${ }^{86}$ plays no role here. If we included a curvature of the dispersion relation for the nanotubes, we would get a correction to the results presented here. However, there is one subtlety hidden in this, because if we consider Coulomb drag between short tubes, where the distribution functions are not relaxed to the Galilean invariant form assumed in Ref. 86, but is instead given by a two- 


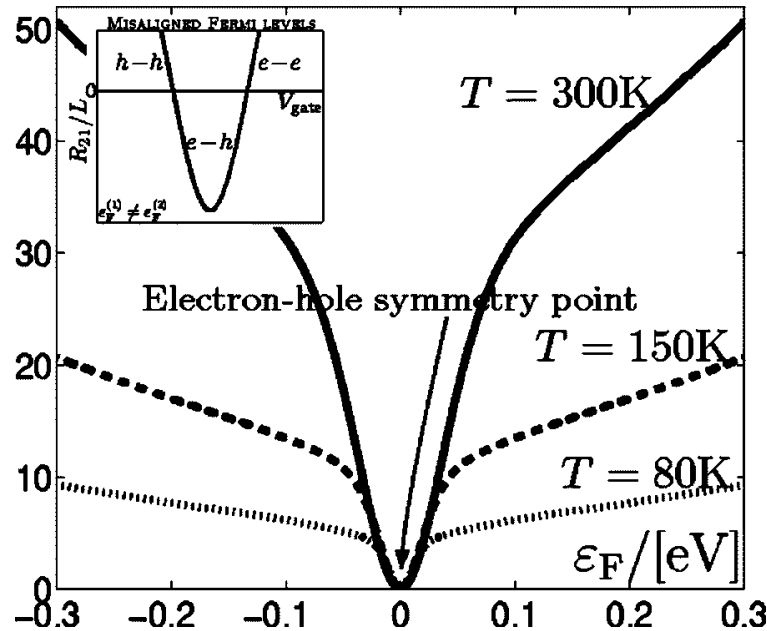

FIG. 4. The transresistance per length $R_{21} / L$ (in $\Omega / \mu \mathrm{m}$ ) as a function of the Fermi level $\varepsilon_{\mathrm{F}}$ (in eV) (e.g., a gate voltage) for equal Fermi levels of the two tubes, $\varepsilon_{\mathrm{F}}=\varepsilon_{\mathrm{F}}^{(1)}=\varepsilon_{\mathrm{F}}^{(2)}$. The temperature is $T$ $=80 \mathrm{~K}$ (dotted), $T=150 \mathrm{~K}$ (dashed), and $T=300 \mathrm{~K}$ (full line). The dip in $R_{21}$ at $\varepsilon_{\mathrm{F}}=0$ reflects the electron-hole symmetry at this point. Inset: A sketch of the situation for misaligned Fermi levels $\varepsilon_{\mathrm{F}}^{(1)}$ $\neq \varepsilon_{\mathrm{F}}^{(2)}$ as a function of gate voltage (see text for details).

step distribution function, the forward scattering does not contribute to the Coulomb drag as shown in Refs. 87 and 88.

Second, we have used a quantum number independent impurity relaxation time $\tau_{i}$ for each subsystem in Eqs. (19) and (20). The mobility $\mu_{\mathrm{Tr}}^{(i)}$ can be shown to be proportional to $\tau_{i}$, i.e. $\mu_{\mathrm{Tr}}^{(i)} \propto \tau_{i}$, from a single subsystem Boltzmann equation [such as Eq. (19)]. Therefore the $F$ function, Eq. (23), is $\tau_{i}$ independent, so the transresistance $R_{21}$ is independent of the impurity relaxation times. So in the quasiballistic regime for large $\tau_{i}$ the transresistance is still formally correct. However, there has been some work on drag between ballistic one-dimensional systems with free-electron-like bands using Boltzmann equations, where almost identical transresistance formula is found. ${ }^{89}$

As a last comment, we note that umklapp scattering is only possible if the tubes are commensurable due to the $\delta_{G_{1}, G_{2}}$ function in Eq. (22) as also found in Ref. 82.

\section{ELECTRON-HOLE SYMMETRY AND COULOMB DRAG}

All nanotubes have an inherited electron-hole symmetry from the graphene band structure for $\varepsilon_{\mathrm{F}}=0$, which intuitively means that there are as many electrons as holes for $\varepsilon_{\mathrm{F}}=0$ (for the precise definition see Ref. 90; for a recent measurement of electron-hole symmetry see Ref. 13). So there will be an equal amount of momentum transfer to (from) the electrons and holes and therefore no voltage difference will arise, i.e., $R_{21}=0$, if one of the subsystems has electron-hole symmetry. Formally, the $F$ function can be seen to vanish at electronhole symmetry by using $f_{-\mu}^{0}(\varepsilon)=1-f_{\mu}^{0}(-\varepsilon)$ (after doing the sum over the band indices), where $\mu$ is the chemical potential. This has also been used to show how $R_{21}$ can change sign. $^{82}$

Therefore, we predict that by varying the Fermi levels (either by gate voltage or doping) a dip (or peak) in $R_{21}$ will appear due to the electron-hole symmetry for all kinds of tube combinations. For two concentric armchair tubes $[(5,5)$ in $(10,10)]$ the transresistance as a function of $\varepsilon_{\mathrm{F}}$ is shown in Fig. 4 (the range of $\varepsilon_{\mathrm{F}}$ is chosen to correspond to typical experiments $\left.{ }^{6,76,77,91-93}\right)$. Note that we use the simplification of having the same Fermi level in the two tubes in the numerical calculation, but the situation for misaligned Fermi levels is sketched in the inset of Fig. 4. As indicated in the inset, we have the following scenario for increasing gate voltage for $\varepsilon_{\mathrm{F}}^{(1)} \neq \varepsilon_{\mathrm{F}}^{(2)}$ : First hole-hole $(h-h)$ scattering, then one subsystem passes electron-hole symmetry, i.e., $R_{21}=0$, afterwards $e-h$ scattering until the other subsystem also passes though the electron-hole symmetry point. The details of the calculation are given below in Sec. VI A.

\section{COULOMB DRAG BETWEEN METALLIC TUBES}

\section{A. Drag between (real) armchair tubes}

Let us begin by calculating the transresistance, Eq. (22), between two concentric real [i.e., $(n, n)]$ armchair nanotubes, which have $|\mathbf{T}|=a$ independent of $n$. The band index $\nu$ is in this case the index $\Pi= \pm 1$ from Eq. (5). To find the $F_{\Pi \Pi^{\prime}}^{(i)}(q, \omega)$ functions, Eq. (23), we need the solutions of $\varepsilon_{k}^{\Pi}$ $-\varepsilon_{k+q}^{\Pi^{\prime}}-\hbar \omega=0$ with the bands, Eq. (5), and remembering that $\varepsilon_{k}^{\Pi}$ should be made $2 \pi /\left|\mathbf{T}_{i}\right|$ periodic by hand (in order to find two solutions and not only one). The signum-function only gives backscattering, which is expressed by step functions. For intraband backscattering $\Pi^{\prime}=\Pi$ we have $g \simeq 1$ [Eq. (16)] and for interband backscattering $\Pi^{\prime}=-\Pi$ we have $|g|^{2}$ $=s_{0}^{2}\left[3(a q)^{2} / 16\right]$ [Eq. (15)] as found in Sec. III A. Therefore the $F$ functions are ${ }^{80}$ for $0<q \leqslant \pi /\left|\mathbf{T}_{i}\right|$ :

$$
\begin{aligned}
F_{--}^{(i)}(q, \omega)= & -C_{F}^{(i)} \theta\left(-\omega+v_{0} q\right) \\
& \times\left\{-\left[f^{0}\left(\varepsilon_{1}\right)-f^{0}\left(-\varepsilon_{2}-\frac{1}{2} k_{0} \hbar v_{0}\right)\right]\right. \\
& \left.+\left[f^{0}\left(\varepsilon_{2}\right)-f^{0}\left(-\varepsilon_{1}-\frac{1}{2} k_{0} \hbar v_{0}\right)\right]\right\},
\end{aligned}
$$

with

$$
\begin{aligned}
\varepsilon_{1}=(\hbar / 2)\left(\omega+v_{0} q-2 v_{0} k_{0}\right) \text { and } \varepsilon_{2}=(\hbar / 2)\left(\omega-v_{0} q+v_{0} k_{0}\right), \\
F_{++}^{(i)}(q, \omega)=-C_{F}^{(i)} \theta\left(-\omega+v_{0} q\right) \\
\times\left\{-\left[f^{0}\left(\widetilde{\varepsilon}_{1}\right)-f^{0}\left(-\widetilde{\varepsilon}_{2}+\frac{1}{2} \hbar v_{0} k_{0}\right)\right]\right. \\
\left.+\left[f^{0}\left(\widetilde{\varepsilon}_{2}\right)-f^{0}\left(-\widetilde{\varepsilon}_{1}+\frac{1}{2} \hbar v_{0} k_{0}\right)\right]\right\}
\end{aligned}
$$

with

$$
\widetilde{\varepsilon}_{1}=(\hbar / 2)\left(\omega+v_{0} q-v_{0} k_{0}\right) \text { and } \widetilde{\varepsilon}_{2}=(\hbar / 2)\left(\omega-v_{0} q+2 v_{0} k_{0}\right),
$$



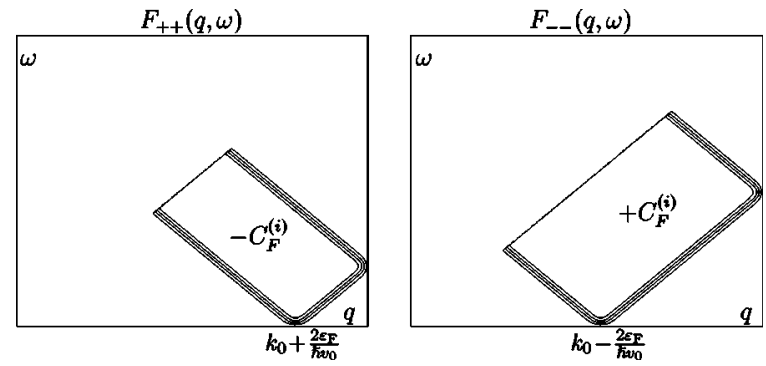

FIG. 5. Contour plot of the $F$ functions for the intraband scattering for $0<q<\pi /|\mathbf{T}|, \varepsilon_{\mathrm{F}}>0$ and the temperature $T=0.1 T_{\mathrm{F}}$. Note the smearing by the Fermi functions due to the temperature on some edges and the sharp edge at $\omega=v_{0} q$ from the step function $\theta\left(-\omega+v_{0} q\right)$

$$
\begin{aligned}
F_{+-}^{(i)}(q, \omega)= & -C_{F}^{(i)} s_{0}^{2} \frac{3(q a)^{2}}{16} \theta\left(\omega-v_{0} q+v_{0} k_{0}\right) \\
& \times \theta\left(-\omega-v_{0} q+2 v_{0} k_{0}\right) \\
& \times\left\{-\left[f^{0}\left(\varepsilon_{1}^{\prime}\right)-f^{0}\left(-\varepsilon_{2}^{\prime}\right)\right]+\left[f^{0}\left(\varepsilon_{2}^{\prime}\right)-f^{0}\left(-\varepsilon_{1}^{\prime}\right)\right]\right\}
\end{aligned}
$$

and

$$
\begin{aligned}
F_{-+}^{(i)}(q, \omega)= & -C_{F}^{(i)} s_{0}^{2} \frac{3(q a)^{2}}{16} \theta\left(-\omega-v_{0} q+v_{0} k_{0}\right) \\
& \times\left\{-\left[f^{0}\left(\varepsilon_{1}^{\prime}\right)-f^{0}\left(-\varepsilon_{2}^{\prime}\right)\right]+\left[f^{0}\left(\varepsilon_{2}^{\prime}\right)-f^{0}\left(-\varepsilon_{1}^{\prime}\right)\right]\right\},
\end{aligned}
$$

where $\varepsilon_{1}^{\prime}=(\hbar / 2)\left(\omega+v_{0} q\right)$ and $\varepsilon_{2}^{\prime}=(\hbar / 2)\left(\omega-v_{0} q\right)$ and we have calculated the common single subsystem prefactor

$$
C_{F}^{(i)} \equiv \frac{\tilde{e}_{i} \tau_{i}}{\hbar^{2} \mu_{\mathrm{Tr}}^{(i)}}=\frac{2 \varepsilon_{\mathrm{F}}+\hbar v_{0} \pi /\left|\mathbf{T}_{i}\right|}{2\left(\hbar v_{0}\right)^{2}} .
$$

It is important to note that the interband $F$ functions, $F_{+-}$and $F_{-+}$, are heavily suppressed compared to the intraband $F$ functions (shown in Fig. 5) by $|g|^{2}=s_{0}^{2}\left[3(a q)^{2} / 16\right]$ of order $\lesssim 10^{-4}$ for backscattering around the Fermi level. Therefore, including the tight-binding states in the Coulomb matrix element and not just in the available phase space for scattering as in Ref. 94 is a very important effect.

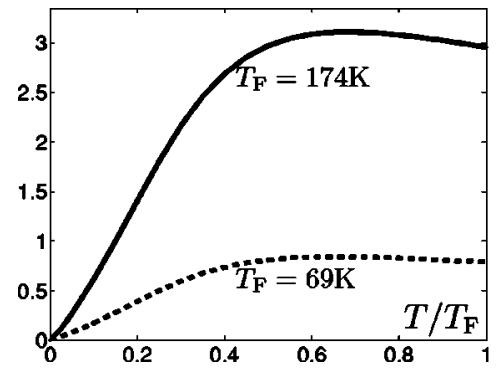

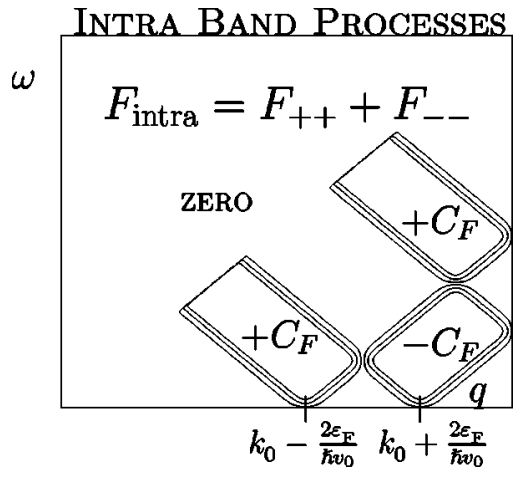

FIG. 6. Contour plot of the $F_{\text {intra }}$ function. $F_{\text {intra }}$ gives the phase space for intraband scattering in (real) armchair tubes. $F_{\text {intra }}$ is seen for $0<q<\pi / a$ and is odd in $q$ and should be repeated periodically with $2 \pi / a$ as a function of $q$.

In real armchair tubes the $\Pi$ index is a parity index in the cylindrical coordinate ${ }^{73,74}$ and therefore the Coulomb matrix element has the property

$$
\begin{aligned}
& \left\langle k_{1}^{\prime} \Pi_{1}^{\prime} k_{2}^{\prime} \Pi_{2}^{\prime}\left|V\left(\mathbf{r}_{1}, \mathbf{r}_{2} \mid\right)\right| k_{1} \Pi_{1} k_{2} \Pi_{2}\right\rangle \\
& \quad=\Pi_{1}^{\prime} \Pi_{2}^{\prime} \Pi_{1} \Pi_{2}\left\langle k_{1}^{\prime} \Pi_{1}^{\prime} k_{2}^{\prime} \Pi_{2}^{\prime}\left|V\left(\mathbf{r}_{1}, \mathbf{r}_{2} \mid\right)\right| k_{1} \Pi_{1} k_{2} \Pi_{2}\right\rangle,
\end{aligned}
$$

i.e., the product of the parity is conserved in the interaction. Since both $\Pi= \pm 1$ have $\mathfrak{m}=0$ there is no angular momentum selection rule, so the only selection rule $\mathcal{J}$ in Eq. (22) is $\mathcal{J}\left(\Pi_{1}, \Pi_{1}^{\prime}, \Pi_{2}, \Pi_{2}^{\prime}\right)=\delta_{\Pi_{1} \Pi_{2}, \Pi_{1}^{\prime} \Pi_{2}^{\prime}}$, which reduces the number of terms by a factor of two. Since $V(q, \Delta \mathfrak{m})$ is parity independent in Eq. (22), then the sum over band indices for $\left|\mathbf{T}_{1}\right|$ $=\left|\mathbf{T}_{2}\right|$ is

$$
\begin{aligned}
& \sum_{\Pi_{1} \Pi_{2} \Pi_{1}^{\prime} \Pi_{2}^{\prime}} F_{\Pi_{1} \Pi_{1}^{\prime}} F_{\Pi_{2} \Pi_{2}^{\prime}} \delta_{\Pi_{1} \Pi_{2}, \Pi_{1}^{\prime} \Pi_{2}^{\prime}} \\
& \quad=\left(F_{++}+F_{--}\right)^{2}+\left(F_{+-}+F_{+-}\right)^{2} \equiv\left(F_{\text {intra }}\right)^{2}+\left(F_{\text {inter }}\right)^{2},
\end{aligned}
$$

which defines the inter- and intraband $F$ functions. $\left(F_{\text {inter }}\right)^{2}$ is of fourth order in $s_{0} q$ and therefore strongly suppressed compared to $F_{\text {intra }}$ even though $F_{\text {inter }}$ has a phase space for smaller $q$ and $\omega . F_{\text {intra }}(q, \omega)$ is shown in Fig. 6 .

We now have all the ingredients of the transresistance $R_{21}$ :

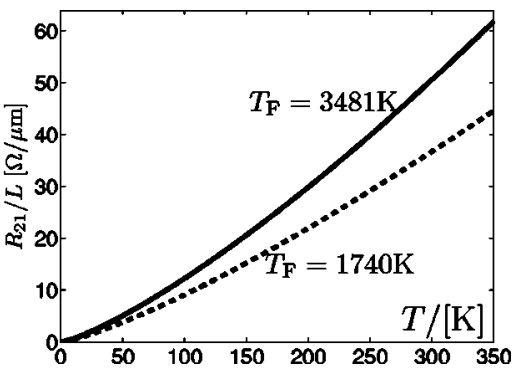

FIG. 7. The transresistance per length $R_{21} / L$ (in units of $\Omega / \mu \mathrm{m}$ ) versus temperature $T$ [or $T / T_{\mathrm{F}}$ (left)]. The curves are obtained from a numerical integration of Eq. (31) for a $(5,5)$ in a $(10,10)$ tube. Curves for four different Fermi levels $\varepsilon_{\mathrm{F}}=\varepsilon_{\mathrm{F}}^{(1)}=\varepsilon_{\mathrm{F}}^{(2)}(\mathrm{i} . e$., gate voltages or dopings) are seen: $\varepsilon_{\mathrm{F}}=0.006 \mathrm{eV}\left(T_{\mathrm{F}}=69 \mathrm{~K}\right)$ (left, dashed line), $\varepsilon_{\mathrm{F}}=0.015 \mathrm{eV}\left(T_{\mathrm{F}}=174 \mathrm{~K}\right)$ (left, solid line), $\varepsilon_{\mathrm{F}}=0.15 \mathrm{eV}\left(T_{\mathrm{F}}=1740 \mathrm{~K}\right)($ right, dashed line) and $\varepsilon_{\mathrm{F}}=0.3 \mathrm{eV}$ (right, solid line). Note the difference in magnitude between the transresistances $R_{21}$. 


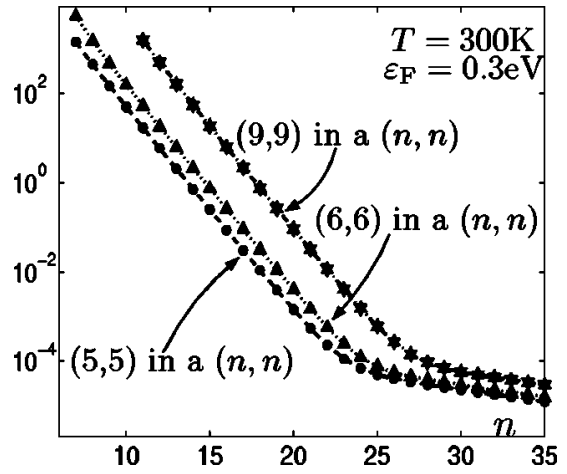

FIG. 8. The transresistance per length $R_{21} / L$ versus radius $(r$ $\propto n)$ for armchair tubes. The different outer and inner armchair tubes are: $\mathrm{A}(5,5)$ in a $(n, n)$ (dots), a $(6,6)$ in a $(n, n)$ (triangles) and a $(9,9)$ in a $(n, n)$ (stars). The radius of the outer tube is: $r$ $=\sqrt{3} a / 2 \pi n$ for a $(n, n)$ tube. Here $T=300 \mathrm{~K}$ and $\varepsilon_{\mathrm{F}}^{(1)}=\varepsilon_{\mathrm{F}}^{(2)}=\varepsilon_{\mathrm{F}}$ $=0.3 \mathrm{eV}$ is used. Note the logarithmic scale.

$$
\begin{aligned}
\frac{R_{21}}{L}= & \frac{\hbar^{2}}{\pi e^{2} n_{1} n_{2} k_{\mathrm{B}} T} \frac{1}{2 \pi r_{1} r_{2}} \int_{0}^{\infty} \frac{d q}{2 \pi} \int_{0}^{\infty} d \omega \frac{\left|V_{12}(q, 0, \omega)\right|^{2}}{\sinh ^{2}\left(\hbar \omega / 2 k_{\mathrm{B}} T\right)} \\
& \times\left\{\left[F_{\text {intra }}(q, \omega)\right]^{2}+\left[F_{\text {inter }}(q, \omega)\right]^{2}\right\} .
\end{aligned}
$$

A numerical integration yields $R_{21}$ as a function of $\varepsilon_{\mathrm{F}}$ and the temperature $T$, shown in Figs. 4 and 7 , respectively. The transresistance per length $R_{21} / L$ is of the order a few $\Omega / \mu \mathrm{m}$. $R_{21}$ is seen to be linear in $T$ for $T \leqq 0.4 T_{\mathrm{F}}$ as also found for free-electron-like bands. ${ }^{95}$ For higher temperatures the transresistance increases or decreases depending on the Fermi level. Numerically, we find a factor of $10^{6}$ difference between the contribution to $R_{21}$ from $F_{\text {inter }}$ and $F_{\text {intra }}$, so we can conclude that the drag is due to the intraband backscattering processes. The largest contribution to the integral is around $q=k_{0} \pm 2 \varepsilon_{\mathrm{F}} / \hbar v_{0}$ (see Fig. 6), which corresponds to umklapp scattering processes around the Fermi level, e.g., $k=k_{0}$ $-\varepsilon_{\mathrm{F}} / \hbar v_{0}$ and $k^{\prime}=-k_{0}+\varepsilon_{\mathrm{F}} / \hbar v_{0}$ so $q=k^{\prime}-k+2 \pi /\left|\mathbf{T}_{i}\right|=k_{0}$ $+2 \varepsilon_{\mathrm{F}} / \hbar v_{0}$.

Note that screening induced by the substrate could change the magnitude of the transresistance a small amount, which could be modeled ${ }^{20}$ by introducing a new dielectric constant $\kappa=\epsilon_{r} \epsilon_{0}$ instead of $\epsilon_{0}$ in Eq. (B15) with $\epsilon_{r}$ about 1 to $3 .{ }^{96}$ For the present case, the magnitude of $R_{21}$ is changed $\lesssim 10 \%$, when $\epsilon_{r}$ is increased from 1 to 3 .

The transresistance depends on the radii of the tubes only via the bare Coulomb interaction Eq. (B15). Figure 8 shows that $R_{21}$ decreases exponentially (for $n \lesssim 25$ ) when keeping the inner armchair tube at a fixed radius and increasing the outer tube radius. For parallel two-dimensional electron gases $R_{21}$ was found to depend on the separation $d$ as ${ }^{55} R_{21}$ $\propto d^{4}$.

\section{B. Drag between armchairlike tubes}

For two general armchairlike tubes, we do not have a parity selection rule and in general $\left|\mathbf{T}_{1}\right| \neq\left|\mathbf{T}_{2}\right|$ as seen in the Table in Appendix A. Therefore we have no selection rules, but all other terms than $F_{\Pi \Pi}^{(1)} F_{\Pi^{\prime} \Pi^{\prime}}^{(2)}$ are of higher order in $\left(s_{0} q\right)^{2}$ and therefore small, i.e.,

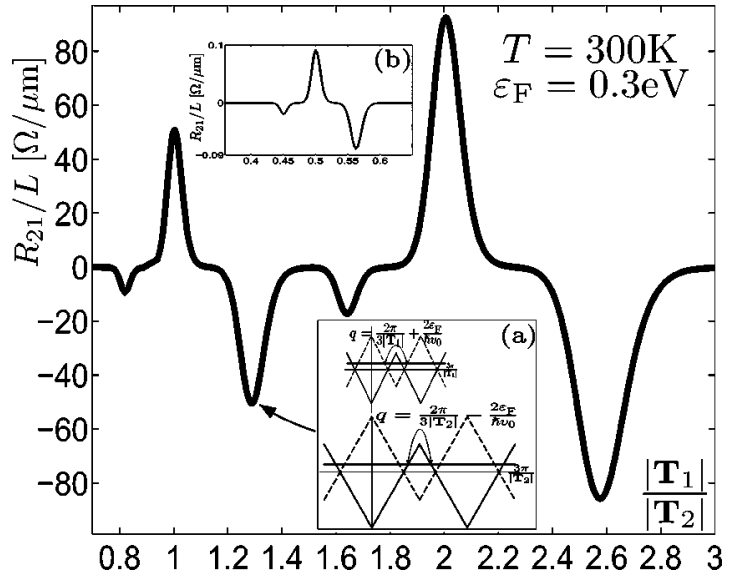

FIG. 9. The transresistance per length $R_{21} / L$ as a function of the ratio of the translational vectors length $\left|\mathbf{T}_{1}\right| /\left|\mathbf{T}_{2}\right|$ for two armchairlike tubes. The peaks corresponding to different scattering processes are seen as explained in the text. Numerically, we use $\left|\mathbf{T}_{2}\right|=a$, radii as for a $(5,5)$ in a $(10,10)$ tube, $T=300 \mathrm{~K}$ and $\varepsilon_{\mathrm{F}}^{(1)}=\varepsilon_{\mathrm{F}}^{(2)}=\varepsilon_{\mathrm{F}}$ $=0.3 \mathrm{eV}$. If the tubes have a different radius, only the magnitude of the peak is changed (see Fig. 8). Inset (a): The scattering processes in tube 1 and 2 leading to the peak at $\left|\mathbf{T}_{1}\right| /\left|\mathbf{T}_{2}\right| \simeq 1.28$. Note that the backscattering processes are electronlike and holelike, respectively, so $R_{21}<0$. Inset (b): Peaks around $\left|\mathbf{T}_{1}\right| /\left|\mathbf{T}_{2}\right|=1 / 2$. Note the difference in scale.

$$
\begin{aligned}
\sum_{\Pi_{1} \Pi_{2} \Pi_{1}^{\prime} \Pi_{2}^{\prime}} F_{\Pi_{1} \Pi_{1}^{\prime}}^{(1)} F_{\Pi_{2} \Pi_{2}^{\prime}}^{(2)} \simeq & F_{++}^{(1)} F_{++}^{(2)}+F_{--}^{(1)} F_{--}^{(2)}+F_{++}^{(1)} F_{--}^{(2)} \\
& +F_{--}^{(1)} F_{++}^{(2)}+O\left(\left(s_{0} q\right)^{2}\right)
\end{aligned}
$$

as for the (real) armchair tube case Eq. (30). The $F_{--}^{(i)}$ and $F_{++}^{(i)}$ are the same as those found in Sec. VI A and shown in Fig. 5 except that $a$ is replaced by $\left|\mathbf{T}_{i}\right|$ (but not in the $g$ factor).

Since $\left|\mathbf{T}_{1}\right|$ and $\left|\mathbf{T}_{2}\right|$ are different (in general), it is harder to conserve (crystal) momentum near the Fermi level for the dominant backscattering process with momentum transfer $q$ $\simeq k_{0}^{(i)} \pm 2 \varepsilon_{\mathrm{F}} / \hbar v_{0}$ with $k_{0}^{(i)}=2 \pi / 3\left|\mathbf{T}_{i}\right|$. However, for some values of $\left|\mathbf{T}_{1}\right|$ and $\left|\mathbf{T}_{2}\right|$ it is possible to conserve momentum near the Fermi level, which gives rise to peaks in $R_{21}$, e.g., at $\left|\mathbf{T}_{1}\right| /\left|\mathbf{T}_{2}\right|=1$ as seen in Fig. 9. The peaks on both sides of $\left|\mathbf{T}_{1}\right| /\left|\mathbf{T}_{2}\right|=1$ are

$$
\left|\frac{\left|\mathbf{T}_{2}\right|-\left|\mathbf{T}_{1}\right|}{\left|\mathbf{T}_{1}\right|\left|\mathbf{T}_{2}\right|}\right|=\frac{6 \varepsilon_{\mathrm{F}}}{\pi \hbar v_{0}},
$$

corresponding to $k_{0}^{(1)} \pm 2 \varepsilon_{\mathrm{F}} / \hbar v_{0}=k_{0}^{(2)} \mp 2 \varepsilon_{\mathrm{F}} / \hbar v_{0}$ [see inset (a) in Fig. 9]. These peaks have $R_{21}<0$, since they correspond to a resonance between a electronlike and a holelike backscattering in the sense that a holelike (electronlike) backscattering takes place in a holelike (electronlike) band with $\operatorname{sgn}\left(v_{k}\right)=-\operatorname{sgn}(k)\left(\operatorname{sgn}\left(v_{k}\right)=\operatorname{sgn}(k)\right)$ in the FBZ. The peaks around $\left|\mathbf{T}_{1}\right| /\left|\mathbf{T}_{2}\right|=\frac{1}{2}$ and 2 are found in the same way by taking the backscattering processes $q \simeq 2 k_{0}^{(i)} \pm 2 \varepsilon_{\mathrm{F}} / \hbar v_{0}$ into account. If the radii of the tubes are different, then the magnitude of $R_{21}$ will change (see Fig. 8), but the signs and positions of the peaks are the same. The peaks are broadened 
by increasing temperature and the positions of the peaks depend on $\varepsilon_{\mathrm{F}}$ as seen, e.g., from Eq. (33) (except for $\left|\mathbf{T}_{1}\right| /\left|\mathbf{T}_{2}\right|=\frac{1}{2}, 1$, and 2). The situation of varying $\left|\mathbf{T}_{1}\right|$ and $\left|\mathbf{T}_{2}\right|$ is similar to varying the densities in the parallel twodimensional (2D) systems. ${ }^{81}$ Note that if we have a tube configuration corresponding to a negative dip in Fig. $9\left(R_{21}\right.$ $<0$ ), then this tube configuration will have a peak instead of a dip as a function of the gate voltage. Summarizing, the Coulomb drag between armchairlike tubes is strongly dependent on the magnitude of the translational vectors $\left|\mathbf{T}_{1}\right|$ and $\left|\mathbf{T}_{2}\right|$ and can lead to both negative and positive transresistance.

\section{Drag between zigzaglike tubes}

Consider the drag between two zigzaglike tubes, where the $\nu$ index is $\xi= \pm 1$ and $\mathfrak{m} \in\left\{\mathfrak{m}_{a}, \mathfrak{m}_{b}\right\}$ from Eq. (4). The backscattering $F$ function, $F_{\xi \mathfrak{m},-\xi \mathfrak{m}^{\prime}}^{(i)}$, has a form similar to $F_{\Pi,-\Pi}^{(i)}$ for armchairlike tubes [Eqs. (26) and (27)], where the important part is the backscattering around small $q$ $\simeq 2 \varepsilon_{\mathrm{F}} / \hbar v_{0}$. This backscattering can be both with $(\Delta \mathfrak{m} \neq 0)$ and without $(\Delta \mathfrak{m}=0)$ exchange of crystal angular momentum with the $g$ factors

$$
|g(\Delta \mathfrak{m}=0)|^{2} \propto\left(s_{0} a q\right)^{2} \text { and }|g(\Delta \mathfrak{m} \neq 0)|^{2} \simeq 1
$$

found in Sec. III A. Since there is crystal angular momentum conservation $^{97}$ it depends on the combination of the zigzaglike tubes (and their $\mathfrak{m}_{a}$ and $\mathfrak{m}_{b}$ ) whether the $\Delta \mathfrak{m} \neq 0$ backscattering is possible or not, so we have two very different cases.

(1) If $\Delta \mathfrak{m} \neq 0$ is not possible, then only $\Delta \mathfrak{m}=0$ backscattering for $q \simeq 2 \varepsilon_{\mathrm{F}} / \hbar v_{0}$ is present, but this is strongly suppressed by the small $g$ factor and so is the drag. So in this case the small wave vector transfer forward scattering (for nonlinearized bands) could become important, but in any case the effect is small. An example is the drag between two (real) metallic zigzag tubes (see Table I).

(2) If $\Delta \mathfrak{m} \neq 0$ is possible, then this process is the dominant, even though there is a small suppression (compared to the $g$ factor) from having $\Delta \mathfrak{m} \neq 0$ in the Fourier transform $V_{12}(q, \Delta \mathfrak{m}, \omega)$, which is smaller the larger $\Delta \mathfrak{m}$. An example is a $(12,15)$ in a $(15,18)$, which has an angular momentum exchange of $\Delta \mathfrak{m}= \pm 1$.

Furthermore, there are no peaks in $R_{21}$ as a function of $\left|\mathbf{T}_{1}\right| /\left|\mathbf{T}_{2}\right|$ as for the armchairlike tubes, since the transferred crystal wave vector $q \simeq \pm 2 \varepsilon_{\mathrm{F}} / \hbar v_{0}$ is independent of $\left|\mathbf{T}_{i}\right|$. From the same principles as used above, we find the drag between zigzaglike and armchairlike tubes to be strongly suppressed.

\section{COMMENTS ON THE DRAG BETWEEN SEMICONDUCTING TUBES}

If the Fermi level for a semiconducting tube is shifted into the conduction (or valence) band, then the drag processes are within a single band (i.e., $\Delta \mathfrak{m}=0$ ) similar to a quadratic band for small tubes, where there are few bands with large separation. Here both the small $q$ forward scattering and the large $q$ backscattering processes will contribute to the drag. We can calculate the $g$ factors in the same way as for the metallic tubes and for intraband scattering they are of order one. However, the magnitude of the backscattering momentum transfer around the Fermi level has to be approximately the same in the two tubes in order to satisfy momentum conservation. In general, this is not the case.

If we deal with larger tubes more bands can come into play and thereby more scattering possibilities appear than captured in the single band quadratic model (see Ref. 70 for a discussion on scattering in larger MWCNT's). This is also the case of larger metallic tubes. Coulomb drag in the quadratic model with more bands (with different angular momentum along the tube) for tubes of semiconducting material is considered in Ref. 98.

\section{SUMMARY}

We have considered the intershell resistance $R_{21}$ originating from the intershell Coulomb interaction neglecting tunneling, i.e., in a Coulomb drag configuration. For any tube combination we predict a dip or peak in $R_{21}$ as a function of gate voltage, which could be experimentally observable. The dip (or peak) is due to the electron-hole symmetry of the carbon nanotube band structure. Whether $R_{21}$ has a dip or peak depends on the sign of $R_{21}$, when both systems have Fermi levels above the electron-hole symmetry point.

The order of magnitude and sign of $R_{21}$ were found to depend crucially on the chirality and Fermi level mismatching of the two tubes. The order of magnitude of $R_{21}$ can reach $\sim 50 \Omega / \mu \mathrm{m}$ under favorable circumstances. The origin of the drastic change in magnitude between different chiralities is the suppressed backscattering due to the Coulomb matrix element between Bloch states combined with the mismatching of wave vector and crystal angular momentum conservation near the Fermi level. The intershell resistance $R_{21}$ was found to be linear in temperature for low temperatures (compared to $T_{\mathrm{F}}$ ), just as for a single quadratic band. To facilitate the analysis, we classified all metallic tubes in two categories: zigzaglike or armchairlike, and described their crystal angular momentum properties.

Throughout the paper, we use Fermi liquid theory to describe the Coulomb drag in the MWCNT's, which gives a benchmark result for comparison to future experiments and Luttinger liquid theories of drag in MWCNT's. The effects considered in this paper should be helpful in interpreting future measurements of the intershell resistance.

\section{ACKNOWLEDGMENTS}

We thank Mads Brandbyge for several very useful discussions on the carbon nanotube band structure and Reinhold Egger for a discussion on the problem of intershell resistance. Furthermore, Laurits Højgaard Olesen is greatly acknowledged for his help on numerical integration and use of MATLAB. Jesper Nygård, Birte Rasmussen, and Peter Bøggild have given several useful comments on the experimental realization of Coulomb drag in multiwall carbon nanotubes. Anna A. Jensen and Morten H. Larsen are acknowledged for 
discussions of an algebraic nature. A helpful comment from T. Vuković is also appreciated.

\section{APPENDIX A: ENERGY BAND STRUCTURE OF THE CARBON NANOTUBES}

We will now give a rather detailed discussion of the band structure of carbon nanotubes, since the intershell Coulomb interaction matrix element depends critically on the Bloch states of the two tubes due to the two atomic unit cell (of a graphite layer) as seen in Sec. III. The carbon nanotube lattice can be thought of as a wrapping (i.e., a conformal mapping) of a graphite layer into a tube. The wrapping is preformed such that the chiral vector $\mathbf{C}=n \mathbf{a}_{1}+m \mathbf{a}_{2}$ becomes the circumferential of the $(n, m)$ nanotube and this determines the lattice completely. ${ }^{99,100}$ [Here $\mathbf{a}_{1}=(a / 2)(\sqrt{3},-1)$ and $\mathbf{a}_{2}$ $=(a / 2)(\sqrt{3}, 1)$ are graphene lattice vectors and $a=\left|\mathbf{a}_{i}\right|$ $=\sqrt{3} a_{\mathrm{c}-\mathrm{c}}$, where $a_{\mathrm{c}-\mathrm{c}}$ is the interatomic distance.]

Any $(n, m)$ nanotube lattice has three symmetries: A discrete translational symmetry along the tube, a discrete rotational symmetry around the tube axis, and a helical symmetry (i.e., a screw operation). These symmetries gives rise to the three corresponding quantum numbers: $k$ (crystal wave vector along the tube), $\mathfrak{m}$ (the crystal angular momentum component along the tube), and $\kappa$ (helical quantum number). Only two of these symmetries (quantum numbers) are needed to label the eigenstates, since the symmetries are not independent. ${ }^{72}$ Conventionally translational symmetry is used to label the states, but this does not use the smallest possible unit cell and can therefore give many bands in the FBZ with the same angular momentum.

Any carbon nanotube can be generated from a primitive two atomic unit cell using only discrete rotations and discrete screw operations and thereby giving (generalized) Bloch states $|\kappa \mathfrak{m}\rangle .^{71,72}$ The advantage of using this method is that each energy band (as a function of $\kappa$ ) has its own crystal angular momentum $\mathfrak{m}$. The discrete rotational symmetry is generated by the vector $\mathbf{C}_{\mathfrak{n}}$ along $\mathbf{C}$ giving the smallest possible rotation leaving the lattice invariant, i.e.,

$$
\mathbf{C}_{\mathfrak{n}}=\frac{n}{\mathfrak{n}} \mathbf{a}_{1}+\frac{m}{\mathfrak{n}} \mathbf{a}_{2}, \quad \text { where } \quad \mathfrak{n}=\operatorname{gcd}(n, m),
$$

i.e., $\mathfrak{n}$ is the greatest common divisor of $n$ and $m$. So a given $(n, m)$ tube has crystal angular momentum $\mathfrak{m} \in\{0,1, \ldots, n$ $-1\}$. The disadvantage of using the symmetry adapted Bloch states $|\kappa \mathfrak{m}\rangle$ is that $\kappa$ is in the direction of the generator $\mathbf{H}$ for the helical symmetry, which in general is different for different chiral vectors.

If we instead use the (often much) larger translational unit cell the states can be labeled by $k \in]-\pi /|\mathbf{T}|, \pi /|\mathbf{T}|]$, where T generates the translational symmetry (the translational vector) and is given by ${ }^{101}$

$$
\mathbf{T}=\frac{(2 m+n) \mathbf{a}_{1}-(2 n+m) \mathbf{a}_{2}}{\operatorname{gcd}(2 m+n, 2 n+m)} .
$$

Since we do not use the primitive unit cell in this case, but a larger translational unit cell, we get a smaller FBZ and thereby more bands in the FBZ than there are crystal angular momentum quantum numbers.
The conventional way to obtain the band structure for a isolated singlewall $(n, m)$ nanotube using the translational unit cell is to apply periodic boundary conditions on the two dimensional graphene tight-binding state ${ }^{102} \psi_{\mathbf{k}}(\mathbf{r})$ along the circumferential $\mathbf{C}$ of the tube, ${ }^{99,100}$ i.e.,

$$
\psi_{\mathbf{k}}(\mathbf{r}+\mathbf{C})=e^{i \mathbf{k} \cdot \mathbf{C}} \psi_{\mathbf{k}}(\mathbf{r})=\psi_{\mathbf{k}}(\mathbf{r}) \Rightarrow \mathbf{k} \cdot \mathbf{C}=2 \pi n_{c},
$$

where $n_{c}$ is an integer in $\{0,1,2, \ldots, \mathcal{N}-1\}$ with

$$
\mathcal{N}=\frac{2\left(n^{2}+m^{2}+n m\right)}{\operatorname{gcd}(2 m+n, 2 n+m)} \geqslant \mathfrak{n}
$$

being the number of (two atomic) graphene unit cells in a translational unit cell. ${ }^{101}$ Thereby $n_{c}$ labels the bands (as a function of $k$ ) using the translational unit cell. One disadvantage of using this larger translational unit cell is, that $n_{c}$ is not the crystal angular momentum, but only related to the actual physical crystal angular momentum $\mathfrak{m}$ by

$$
n_{c}=\mathfrak{m}(\bmod \mathfrak{n})
$$

Furthermore, we can connect the description of the band structure using the primitive unit cell and the translational unit cell by $\kappa=\mathbf{k} \cdot \mathbf{H}$, i.e., $\kappa$ depends on both $k$ and $n_{c}{ }^{72} \mathrm{An}$ example is given in Fig. 10.

To do a tight-binding calculation for graphene ${ }^{102}$ it is essential that the unit cell of graphene has two atoms, so the tight-binding state (Wannier decomposition of the eigenstate) has two components: ${ }^{103}$

$$
\psi_{\mathbf{k}}(\mathbf{r})=\frac{1}{\sqrt{N}} \sum_{\mathbf{R}} e^{i \mathbf{k} \cdot \mathbf{R}}\left[\alpha_{\mathbf{k}} \Psi(\mathbf{r}-\mathbf{R})+\beta_{\mathbf{k}} \Psi(\mathbf{r}-\mathbf{R}-\mathbf{d})\right]
$$

where $\Psi$ is a $2 p_{z}$ orbital (Wannier function) localized at each atom, $\mathbf{R}=n_{1} \mathbf{a}_{1}+n_{2} \mathbf{a}_{2}\left(n_{1}, n_{2} \in \mathbb{Z}\right)$ are lattice vectors, $N$ is the number of unit cells in the layer, $\mathbf{d}=\frac{1}{3}\left(\mathbf{a}_{1}+\mathbf{a}_{2}\right)$ is the vector between the two atoms in the unit cell and $\alpha_{\mathbf{k}}, \beta_{\mathbf{k}}$ are functions to be determined by the tight-binding calculation. To find the energy we insert $\psi_{\mathbf{k}}(\mathbf{r})$ in $H \psi_{\mathbf{k}}(\mathbf{r})=\varepsilon_{\mathbf{k}} \psi_{\mathbf{k}}(\mathbf{r})$ and obtain a $2 \times 2$ matrix equation:

$$
\left(\begin{array}{ll}
H_{11} & H_{12} \\
H_{21} & H_{22}
\end{array}\right)\left(\begin{array}{c}
\alpha_{\mathbf{k}} \\
\beta_{\mathbf{k}}
\end{array}\right)=\varepsilon_{\mathbf{k}}\left(\begin{array}{ll}
S_{11} & S_{12} \\
S_{21} & S_{22}
\end{array}\right)\left(\begin{array}{c}
\alpha_{\mathbf{k}} \\
\beta_{\mathbf{k}}
\end{array}\right),
$$

where $H_{i j}, S_{i j}$ are the overlap integrals with and without the Hamiltonian found in the nearest neighbor tight-binding approximation to be

$$
\begin{gathered}
H_{12}=-\gamma_{0}\left(1+e^{-i \mathbf{k} \cdot \mathbf{a}_{1}}+e^{-i \mathbf{k} \cdot \mathbf{a}_{2}}\right) \equiv-\gamma_{0} \Upsilon(\mathbf{k}), \\
S_{12}=s_{0} \Upsilon(\mathbf{k}), \quad S_{i i}=1, \quad H_{i i}=\varepsilon_{0},
\end{gathered}
$$

where the value of the overlap integral is $\gamma_{0} \simeq 3 \mathrm{eV}$, the overlap of the orbitals are ${ }^{101} s_{0} \sim 0.1$ and $\varepsilon_{0}$ is the energy of the orbital, which is set to zero. Here the convention $\gamma_{0}, s_{0}$ $>0$ is used and note that others use slightly different values (e.g., $\left.\gamma_{0} \simeq 2.5-3.1\right) .{ }^{104-107}$ By a diagonalization of Eq. (A6) we find $[$ for $Y(\mathbf{k}) \neq 0$ ] 

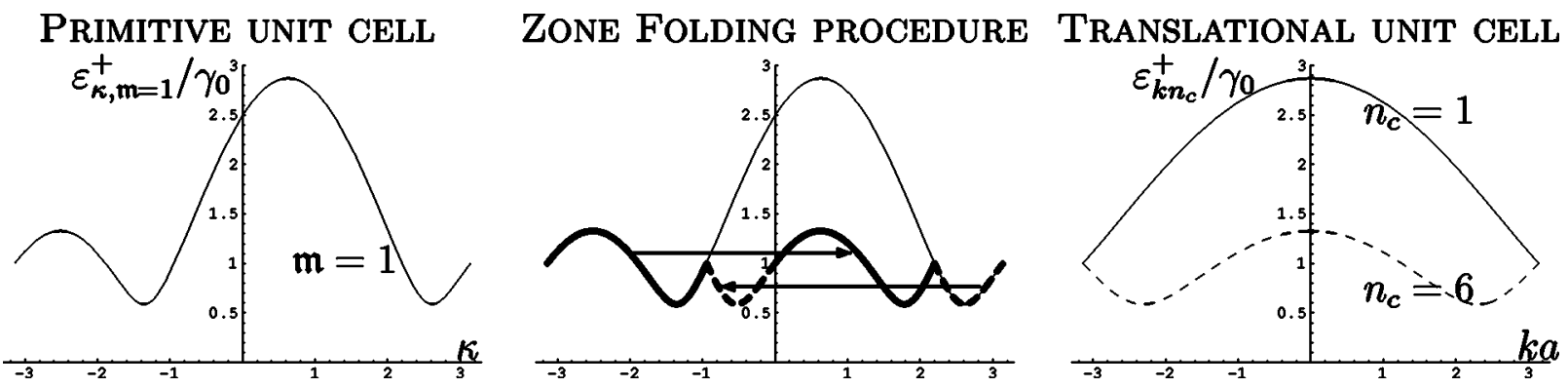

FIG. 10. Left: The $\mathfrak{m}=1$ band for a $(5,5)$ tube in the FBZ of the primitive unit cell as a function of $\kappa \in]-\pi, \pi]$. Center: The $\mathfrak{m}=1$ band is pushed into the smaller FBZ of the translational unit cell by using $\kappa=k a / 2+n_{c} \pi / 5$, with $n_{c}=1$ and $n_{c}=6$, since $\mathfrak{m}=1$. Note that the band is symmetrical around $\pi / 5$, since $\mathfrak{m}=1$. Right: The band structure for the translational unit cell. Both bands have crystal angular momentum $\mathfrak{m}=1$, but indices $n_{c}=1$ and $n_{c}=6$.

$$
\varepsilon_{\mathbf{k}}^{ \pm}= \pm \gamma_{0}|\Upsilon(\mathbf{k})|, \quad\left(\begin{array}{c}
\alpha_{\mathbf{k}} \\
\beta_{\mathbf{k}}
\end{array}\right)_{ \pm}=\frac{1}{\sqrt{2}}\left(\begin{array}{c}
\mp \frac{\Upsilon(\mathbf{k})}{|\Upsilon(\mathbf{k})|} \\
1
\end{array}\right)
$$

where we have neglected $s_{0}$ in the energy (but not in the eigenstate). By inserting $\mathbf{k}$ decomposed along the tube (T) and around the tube $(\mathbf{C}): \mathbf{k}=k \mathbf{T} /|\mathbf{T}|+k_{c} \mathbf{C} /|\mathbf{C}|$ with $k_{c}$ $=(2 \pi /|\mathbf{C}|) n_{c}$, one obtains the band structure for nanotube labeled by $k$ and $n_{c}$. Essentially the same tight-binding calculation can be done using only the helical and rotational symmetry as in Ref. 71 and the same result is found, when we use $\kappa=\mathbf{k} \cdot \mathbf{H}$ and Eq. (A4) to convert between the quantum numbers ${ }^{108}$ (see Fig. 10).

The Fermi level is at $\varepsilon_{\mathrm{F}}=0$, since half of the states $\left(2 p_{z}\right.$ orbitals) are filled. By doping and/or a gate voltage the Fermi level can be moved about $\sim \pm 0.4 \mathrm{eV} .{ }^{6,76,77,91-93}$ Furthermore note that graphene has electron-hole symmetry ${ }^{90}$ for $\varepsilon_{\mathrm{F}}=0$ and therefore so does any $(n, m)$ carbon nanotube.

\section{The linearized band structure}

We are only interested in the transport properties of nanotubes and therefore expand $\Upsilon(\mathbf{k})$ around the Fermi level $\varepsilon_{\mathrm{F}}$ $=0$, i.e., around the two zeros ${ }^{109}$ of $Y(\mathbf{k})$,

$$
\mathbf{K}_{\mathrm{s}}=\frac{2 \pi}{a}\left(\frac{1}{\sqrt{3}}, \mathrm{~s} \frac{1}{3}\right) \quad(\mathrm{s}= \pm 1)
$$

and obtain

$$
\boldsymbol{Y}\left(\mathbf{K}_{\mathrm{\varsigma}}+\mathfrak{K}\right) \simeq \frac{\sqrt{3} a}{2}\left(i \mathfrak{K}_{x}+\varsigma \mathfrak{K}_{y}\right),
$$

where we have introduced the deviation from $\mathbf{K}_{\mathrm{s}}$ by $\mathfrak{K} \equiv \mathbf{k}-\mathbf{K}_{\mathrm{s}}$. Note that $\left|\mathrm{Y}\left(\mathbf{K}_{\mathrm{s}}+\mathfrak{K}\right)\right| \simeq(\sqrt{3} a / 2)|\mathfrak{K}|$ usable in Eq. (A9). Furthermore, note that we do not expand $Y$ around each individual $\varepsilon_{\mathrm{F}} \neq 0$ used, but around $\varepsilon_{\mathrm{F}}=0$, since this preserves the electron-hole symmetry of the band structure. By inserting $\mathfrak{K}=\mathfrak{K}_{T} \mathbf{T} /|\mathbf{T}|+\mathfrak{K}_{C} \mathbf{C} /|\mathbf{C}|$ into the periodic boundary condition, Eq. (A3), the energy is found to be

$$
\varepsilon_{\mathfrak{K}_{T}, n_{c}}^{ \pm}= \pm \frac{2 \hbar v_{0}}{D} \sqrt{\left(\frac{\mathfrak{K}_{T} D}{2}\right)^{2}+\left(n_{c}-\frac{(n+m)+\varsigma \frac{1}{3}(m-n)}{2}\right)^{2}},
$$

where

$$
D=\frac{a \sqrt{n^{2}+m^{2}+m n}}{\pi}
$$

is the diameter and $v_{0}=\sqrt{3} \gamma_{0} a / 2 \hbar$ is the value of the velocity in all metallic tubes.

\section{Unified picture of metallic tubes: armchairlike and zigzaglike tubes}

We will now show using the linearized $Y$, Eq. (A10), that all metallic tubes are either zigzaglike or armchairlike and define the precise meaning of this. If $(n-m) / 3 \in \mathbb{Z}$ the $(n, m)$ tube is metallic and has four crossings of the Fermi level found from Eq. (A11): two [the \pm in Eq. (A11)] for $n_{c}^{\mathrm{s}=+1}=(2 m+n) / 3$ and two $( \pm)$ for $n_{c}^{\mathrm{s}=-1}=(2 n+m) / 3$ (i.e., $\mathfrak{K}_{C}=0$ ). This gives the energy and eigenstates for the bands crossing the Fermi level:

$$
\begin{gathered}
\varepsilon_{\mathfrak{K}_{T}, n_{c}^{\varsigma}}^{\xi}=\xi \hbar v_{0} \mathfrak{K}_{T}, \\
\left(\begin{array}{c}
\alpha \\
\beta
\end{array}\right)_{\xi, \varsigma}=\frac{1}{\sqrt{2}}\left(\begin{array}{c}
-\xi \frac{i(m-n)-\varsigma \sqrt{3}(n+m)}{2 \sqrt{n^{2}+m^{2}+m n}} \\
1
\end{array}\right),
\end{gathered}
$$

where $\xi= \pm 1$. This is found by inserting the linearized $Y$, Eq. (A10), into Eq. (A9) and using $\mathfrak{K}_{C}=0$. By doing this straightforwardly, we get $\operatorname{sgn}\left(\mathfrak{K}_{T}\right)$ in $\alpha$ and $\varepsilon \propto \pm\left|\mathfrak{K}_{T}\right|$, but we require continuity of the states [across the $Y=0$ point where Eq. (A9) was not valid] and remove the signum-function and thereby also the absolute value, i.e., the metallic linear bands cross the Fermi level $\left(\varepsilon_{\mathrm{F}}=0\right)$. Note that $\alpha$ and $\beta$ are independent of $\mathfrak{K}_{T}$ and thereby $k$ (to first order in $k$ ), which turns out to be important in the Coulomb matrix element. The energy bands cross the Fermi level $\left(\varepsilon_{\mathrm{F}}=0\right)$ at $\mathfrak{K}_{T}=0$ and since $k=\mathbf{k} \cdot \mathbf{T} /|\mathbf{T}|=\mathfrak{K}_{T}+\mathbf{K}_{\mathrm{s}} \cdot \mathbf{T} /|\mathbf{T}|$ the crossing of $\varepsilon_{\mathrm{F}}=0$ as a function of $k$ is at 


$$
\mathbf{K}_{\mathrm{s}} \cdot \frac{\mathbf{T}}{|\mathbf{T}|}= \begin{cases}\frac{-2 \pi n}{\operatorname{gcd}(2 n+m, 2 m+n)|\mathbf{T}|} & \text { for } \mathrm{s}=+1 \\ \frac{2 \pi m}{\operatorname{gcd}(2 n+m, 2 m+n)|\mathbf{T}|} & \text { for } \mathrm{s}=-1\end{cases}
$$

which are either both at $k=0$ (double degenerate, $n_{c}^{\varsigma= \pm 1}$ ) or $k= \pm 2 \pi / 3|\mathbf{T}|$ (nondegenerate) for $k$ in the FBZ, $-\pi /|\mathbf{T}| \leqslant k$ $\leqslant \pi /|\mathbf{T}|$ (see Ref. 80 for details). Furthermore, we have the following connection between the crossing of $\varepsilon_{\mathrm{F}}=0$ and the crystal angular momentum of the bands crossing: ${ }^{110}$ If the bands are crossing $\varepsilon_{\mathrm{F}}=0$ at $k=0$, then the two double degenerate crosses have different nonzero angular momentum,

$$
\mathfrak{m}_{a}=\frac{2 n+m}{3}(\bmod \mathfrak{n}), \quad \mathfrak{m}_{b}=\frac{2 m+n}{3}(\bmod \mathfrak{n})
$$

and $\mathfrak{m}_{a} \neq \mathfrak{m}_{b}$. If, on the other hand, the crossing is at $k$ $= \pm 2 \pi / 3|\mathbf{T}|$, then both crosses have $\mathfrak{m}_{a}=\mathfrak{m}_{b}=0$. This makes it possible to divide all metallic tubes into either armchairlike or zigzaglike tubes (see Fig. 2) with the following bands crossing the Fermi level $\left(\varepsilon_{\mathrm{F}}=0\right)$ :

$$
\text { zigzaglike: } \varepsilon_{k \mathfrak{m}}^{\xi}=\xi \hbar v_{0} k, \quad \mathfrak{m} \in\left\{\mathfrak{m}_{a}, \mathfrak{m}_{b}\right\},
$$

armchairlike: $\varepsilon_{k}^{\Pi}=-\Pi \hbar v_{0}\left(|k|-k_{0}\right), \quad \mathfrak{m}=0, \quad(\mathrm{~A} 17)$

where $k_{0}=2 \pi / 3|\mathbf{T}|, \quad \xi= \pm 1, \quad \Pi= \pm 1, \quad$ and $\left.k \in\right]-\pi /|\mathbf{T}|$, $\pi /|\mathbf{T}|$ ]. The translational vector $\mathbf{T}$ (and $|\mathbf{T}|$ ) is different for different metallic tubes independent of the type. Note that the armchairlike bands are in general not connected in the way modelled by Eq. (A17) [consider, e.g., a $(7,4)$ tube], but since they have the same angular momentum $\mathfrak{m}=0$ we connect the bands in this way for convenience. For scattering between the bands we will, however, consider the bands as four bands as we saw in Sec. III. Examples of zigzaglike and armchairlike tubes are found in Table I.
For a (real) armchair $(n, n)$ tube the $\Pi$ index in Eq. (A17) is the parity in the angular coordinate in cylindrical coordinates $^{73,74}$ and the states are $\left(\begin{array}{c}\alpha \\ \beta\end{array}\right)=(1 / \sqrt{2})\left(\begin{array}{c}\Pi \\ 1\end{array}\right)$ to all orders in $k$ (in the nearest-neighbor tight-binding approximation). Results similar to the ones obtained from the linearized $\mathbf{Y}$, Eq. (A10), can by found by using the $\mathbf{k} \cdot \mathbf{p}$ approximation; ${ }^{111}$ however, this does not reveal the crystal angular momentum.

\section{APPENDIX B: SCREENING IN THE RPA APPROACH INCLUDING THE BAND STRUCTURE}

In this appendix, we will calculate the screened Coulomb potential in the RPA in order to include both static and dynamical screening effects in the Coulomb drag, which have been seen to be important perviously for bilayer systems. ${ }^{55,56,81}$ The Dyson equation for the screened potential in real and frequency space is

$$
\begin{aligned}
V\left(\mathbf{r}_{1}, \mathbf{r}_{2}, \omega\right)= & V^{0}\left(\left|\mathbf{r}_{1}-\mathbf{r}_{2}\right|\right)+\int \mathrm{d} \mathbf{r} \int \mathrm{d} \mathbf{r}^{\prime} V^{0}\left(\left|\mathbf{r}_{1}-\mathbf{r}\right|\right) \\
& \times \chi^{0}\left(\mathbf{r}, \mathbf{r}^{\prime}, \omega\right) V\left(\mathbf{r}^{\prime}, \mathbf{r}_{2}, \omega\right),
\end{aligned}
$$

where the noninteracting polarizability is

$$
\chi^{0}\left(\mathbf{r}, t, \mathbf{r}^{\prime}, t^{\prime}\right)=-i \theta\left(t-t^{\prime}\right)\left\langle\left[\hat{\rho}(\mathbf{r}, t), \hat{\rho}\left(\mathbf{r}^{\prime}, t^{\prime}\right)\right]\right\rangle_{0},
$$

where $\hat{\rho}(\mathbf{r}, t)$ is the density operator in the interaction picture and the average $\langle\cdots\rangle_{0}$ is taken for noninteracting particles. By writing the density operator by the help of a complete set of quantum states $\left\{\varphi_{\eta}(\mathbf{r})\right\}$ we find the polarizability to be

$$
\begin{aligned}
& \chi^{0}\left(\mathbf{r}, \mathbf{r}^{\prime}, \omega\right) \\
& =\sum_{\eta \eta^{\prime}} \frac{f^{0}\left(\varepsilon_{\eta}\right)-f^{0}\left(\varepsilon_{\eta^{\prime}}\right)}{\varepsilon_{\eta}-\varepsilon_{\eta^{\prime}}-\omega+i 0^{+}} \varphi_{\eta}^{*}(\mathbf{r}) \varphi_{\eta^{\prime}}^{*}\left(\mathbf{r}^{\prime}\right) \varphi_{\eta^{\prime}}(\mathbf{r}) \varphi_{\eta}\left(\mathbf{r}^{\prime}\right) \\
& \equiv \sum_{\eta \eta^{\prime}} \widetilde{\chi}_{\eta, \eta^{\prime}}^{0}(\omega) \varphi_{\eta}^{*}(\mathbf{r}) \varphi_{\eta^{\prime}}^{*}\left(\mathbf{r}^{\prime}\right) \varphi_{\eta^{\prime}}(\mathbf{r}) \varphi_{\eta}\left(\mathbf{r}^{\prime}\right)
\end{aligned}
$$

TABLE I. Examples of armchairlike (AL) and zigzaglike (ZL) metallic tubes, i.e., all kinds of metallic tubes. For the AL tubes the difference in the length of the translational vector $\mathbf{T}$ and the diameter $D$ is seen and for the ZL tubes we note the variety of the crystal angular momentum $\mathfrak{m}_{a}=[(2 n+m) / 3](\bmod \mathfrak{n})$ and $\mathfrak{m}_{b}=[(2 m+n) / 3](\bmod \mathfrak{n})$ of the bands crossing the Fermi level $\left(\varepsilon_{\mathrm{F}}=0\right)$. Numerically, it turns out that $\left|\mathfrak{m}_{a}-\mathfrak{m}_{b}\right|=1$ for most of the ZL tubes, but there are other cases such as the $(12,24)$ tube. Remember that $\mathfrak{n}=\operatorname{gcd}(n, m)$ and $a=\left|\mathbf{a}_{i}\right|$.

\begin{tabular}{ccccccc}
\hline \hline Chirality & Type & $\mathfrak{m}_{a}$ & $\mathfrak{m}_{b}$ & $\mathfrak{n}$ & $|\mathbf{T}| / a$ & $D / a$ \\
\hline$(n, n)$ & $\mathrm{AL}$ & 0 & 0 & $n$ & 1 & $\sqrt{3} n / \pi$ \\
$(7,4)$ & $\mathrm{AL}$ & 0 & 0 & 1 & $\sqrt{31}$ & $\sqrt{93} / \pi$ \\
$(15,6)$ & $\mathrm{AL}$ & 0 & 0 & 3 & $\sqrt{13}$ & $3 \sqrt{39} / \pi$ \\
$(8,23)$ & $\mathrm{AL}$ & 0 & 0 & 1 & $\sqrt{259}$ & $\sqrt{777} / \pi$ \\
$(10,25)$ & $\mathrm{AL}$ & 0 & 0 & 5 & $\sqrt{13}$ & $5 \sqrt{39} / \pi$ \\
$(n, 0)$ & $\mathrm{ZL}$ & $2 n / 3$ & $n / 3$ & $n$ & $\sqrt{3}$ & $n / \pi$ \\
$(9,6)$ & $\mathrm{ZL}$ & 2 & 1 & 3 & $\sqrt{57}$ & $3 \sqrt{19} / \pi$ \\
$(6,21)$ & $\mathrm{ZL}$ & 2 & 1 & 3 & $\sqrt{201}$ & $3 \sqrt{67} / \pi$ \\
$(18,12)$ & $\mathrm{ZL}$ & 4 & 2 & 6 & $\sqrt{57}$ & $6 \sqrt{19} / \pi$ \\
$(12,24)$ & $\mathrm{ZL}$ & 4 & 8 & 12 & $\sqrt{21}$ & $12 \sqrt{7} / \pi$ \\
\hline \hline
\end{tabular}


where $0^{+}$is a positive infinitesimal, $f^{0}(\varepsilon)$ is the Fermi function and $\widetilde{\chi}_{\eta, \eta^{\prime}}^{0}(\omega)$ was introduced. To find the Coulomb matrix element we insert the RPA equation (B1) into

$$
\begin{aligned}
& \left\langle 1^{\prime} 2^{\prime}\left|V\left(\mathbf{r}_{1}, \mathbf{r}_{2}, \omega\right)\right| 12\right\rangle \\
& \quad=\int \mathrm{d} \mathbf{r}_{1} \int \mathrm{d} \mathbf{r}_{2} \varphi_{1^{\prime}}^{*}\left(\mathbf{r}_{1}\right) \varphi_{2^{\prime}}^{*}\left(\mathbf{r}_{2}\right) V\left(\mathbf{r}_{1}, \mathbf{r}_{2}, \omega\right) \varphi_{1}\left(\mathbf{r}_{1}\right) \varphi_{2}\left(\mathbf{r}_{2}\right)
\end{aligned}
$$

and get

$$
\begin{aligned}
& \left\langle 1^{\prime} 2^{\prime}\left|V\left(\mathbf{r}_{1}, \mathbf{r}_{2}, \omega\right)\right| 12\right\rangle \\
& =\left\langle 1^{\prime} 2^{\prime}\left|V^{0}\left(\left|\mathbf{r}_{1}-\mathbf{r}_{2}\right|\right)\right| 12\right\rangle \\
& \quad+\sum_{\eta \eta^{\prime}} \widetilde{\chi}_{\eta, \eta^{\prime}}^{0}(\omega)\left\langle 1^{\prime} \eta\left|V^{0}\left(\left|\mathbf{r}_{1}-\mathbf{r}\right|\right)\right| 1 \eta^{\prime}\right\rangle
\end{aligned}
$$

$$
\times\left\langle\eta^{\prime} 2^{\prime}\left|V\left(\mathbf{r}^{\prime}, \mathbf{r}_{2}, \omega\right)\right| \eta 2\right\rangle .
$$

This equation can be used for any set of quantum states and in particular for the metallic states for nanotubes, so $\eta$ is the set of indices $(i, k, \xi, \varsigma, \sigma)$, where $i=1,2$ is the tube index, $\sigma$ is the spin and remember that $\mathrm{s}$ determines the angular momentum $\mathfrak{m}$. The screened and unscreened matrix elements, Eqs. (11) and (12), can now be inserted into Eq. (B5) to get the screened matrix element. Doing this, we observe that $g_{1}\left(k_{1} \varsigma_{1} \xi_{1}, k_{1}^{\prime} \varsigma_{1}^{\prime} \xi_{1}^{\prime}\right) g_{2}\left(k_{2} \varsigma_{2} \xi_{2}, k_{2}^{\prime} \varsigma_{2}^{\prime} \xi_{2}^{\prime}\right)$ is a common factor, which simplifies the result. To simplify further, we use that $g_{i}$ and $\widetilde{\chi}_{i}^{0}$ are periodic in the reciprocal lattice $G_{i}$ for subsystem $i, g\left(\eta, \eta^{\prime}\right)=g^{*}\left(\eta^{\prime}, \eta\right)$ and introduce $q_{i} \equiv k_{i}^{\prime}-k_{i}, \Delta \mathfrak{m}_{i} \equiv \mathfrak{m}_{i}^{\prime}$ $-\mathfrak{m}_{i}$ and

$$
\mathcal{W}_{i_{1} i_{2}}\left(q_{1}, \Delta \mathfrak{m}_{1}, q_{2}, \Delta \mathfrak{m}_{2}, \omega\right) \equiv \sum_{G_{i_{1}}, G_{i_{2}}} \sum_{u_{i_{1}}, u_{i_{2}} \in Z} V\left(q_{1}+G_{i_{1}}, \Delta \mathfrak{m}_{1}+\mathfrak{n}_{i_{1}} u_{i_{1}}, q_{2}+G_{i_{2}}, \Delta \mathfrak{m}_{2}+\mathfrak{n}_{i_{2}} u_{i_{2}}, r_{i_{1}}, r_{i_{2}}\right),
$$

where $i_{1}, i_{2}$ are tube indices and $u_{i_{1}}, u_{i_{2}}$ are integers. Equivalently we introduce $\mathcal{W}_{i_{1} i_{2}}^{0}$ for the sum over $V^{0}$ (without the $g$ 's and the $1 / 2 \pi L$ factor). So Eq. (B5) becomes

$$
\begin{aligned}
& \mathcal{W}_{i_{1} i_{2}}\left(q_{i_{1}}, \Delta \mathfrak{m}_{i_{1}}, q_{i_{2}}, \Delta \mathfrak{m}_{i_{2}}, \omega\right) \\
&= 2 \pi L \mathcal{W}_{i_{1} i_{2}}^{0}\left(q_{i_{1}}, \Delta \mathfrak{m}_{i_{1}}, q_{i_{2}}, \Delta \mathfrak{m}_{i_{2}}\right) \\
&+\sum_{G_{i_{1}} u_{i_{1}}} \sum_{i} V^{0}\left(q_{i_{1}}+G_{i_{1}}, \Delta \mathfrak{m}_{i_{1}}+\mathfrak{n}_{i_{1}} u_{i_{1}}, r_{i_{1}}, r_{i}\right) \\
& \times \chi_{\mathrm{eff}, i}^{0}\left(q_{i_{1}}+G_{i_{1}}, \Delta \mathfrak{m}_{i_{1}}+\mathfrak{n}_{i_{1}} u_{i_{1}}, \omega\right) \\
& \times \mathcal{W}_{i_{i_{2}}}\left(q_{i_{1}}+G_{i_{1}}, \Delta \mathfrak{m}_{i_{1}}+\mathfrak{n}_{i_{1}} u_{i_{1}}, q_{i_{2}}, \Delta \mathfrak{m}_{i_{2}}, \omega\right),
\end{aligned}
$$

which has a matrix structure in the reciprocal lattice and in $i$ and the effective polarization is

$$
\begin{aligned}
\chi_{\mathrm{eff}, i}^{0}(q, \Delta \mathfrak{m}, \omega)= & \frac{2}{2 \pi L} \sum_{k \varsigma} \sum_{\xi \xi^{\prime}} \widetilde{\chi}_{i}^{0}\left(k \xi \varsigma, k+q \xi^{\prime} \boldsymbol{s}^{\prime}, \omega\right) \\
& \times\left|g_{i}\left(k, \xi, \boldsymbol{\varsigma} ; k+q, \xi^{\prime}, \boldsymbol{s}^{\prime}\right)\right|^{2},
\end{aligned}
$$

where $s^{\prime}$ is chosen such that $\mathfrak{m}^{\prime}=\mathfrak{m}+\Delta \mathfrak{m}$. Note that $\widetilde{\chi}^{0}$ is diagonal in the tube index $i$, since we do not include tunnelling between the tubes. In order to find the screened intershell Coulomb interaction we truncate Eq. (B7) and only include the $G_{i_{1}}=0$ and $u_{i_{1}}=0$ term in the sum, which gives us a $2 \times 2$ matrix equation (in $i$ ) to find $\mathcal{W}_{12}$, and therefore the screened Coulomb matrix element is

$$
\begin{aligned}
& \left\langle k_{1}^{\prime} \mathfrak{m}_{1}^{\prime} \xi_{1}^{\prime}, k_{2}^{\prime} \mathfrak{m}_{2}^{\prime} \xi_{2}^{\prime}\left|V\left(\mathbf{r}_{1}, \mathbf{r}_{2}, \omega\right)\right| k_{1} \mathfrak{m}_{1} \xi_{1}, k_{2} \mathfrak{m}_{2} \xi_{2}\right\rangle \\
& =\frac{1}{2 \pi L} g_{1}\left(k_{1} \varsigma_{1} \xi_{1}, k_{1}^{\prime} \varsigma_{1}^{\prime} \xi_{1}^{\prime}\right) g_{2}\left(k_{2} \varsigma_{2} \xi_{2}, k_{2}^{\prime} \boldsymbol{s}_{2}^{\prime} \xi_{2}^{\prime}\right) \\
& \quad \times \sum_{G_{1}, G_{2}} \sum_{u_{1}, u_{2}} \frac{V^{0}\left(k_{1}^{\prime}-k_{1}+G_{1}, \mathfrak{m}_{1}^{\prime}-\mathfrak{m}_{1}+\mathfrak{n}_{1} u_{1}, r_{1}, r_{2}\right)}{\epsilon_{12}\left(k_{1}^{\prime}-k_{1}, \mathfrak{m}_{1}^{\prime}-\mathfrak{m}_{1}, \omega\right)} \\
& \quad \times \delta_{k_{1}+k_{2}, k_{1}^{\prime}+k_{2}^{\prime}+G_{1}+G_{2}} \delta_{\mathfrak{m}_{1}^{\prime}+\mathfrak{m}_{2}^{\prime}+\mathfrak{n}_{1} u_{1}, \mathfrak{m}_{1}+\mathfrak{m}_{2}+\mathfrak{n}_{2} u_{2},}
\end{aligned}
$$

with

$$
\begin{aligned}
\epsilon_{12}(q, \Delta \mathfrak{m}, \omega)= & {\left[1-\chi_{\mathrm{eff}, 1}^{0}(q, \Delta \mathfrak{m}, \omega) V^{0}\left(q, \Delta \mathfrak{m}, r_{1}, r_{1}\right)\right] } \\
& \times\left[1-\chi_{\mathrm{eff}, 2}^{0}(q, \Delta \mathfrak{m}, \omega) V^{0}\left(q, \Delta \mathfrak{m}, r_{2}, r_{2}\right)\right] \\
& -\chi_{\mathrm{eff}, 1}^{0}(q, \Delta \mathfrak{m}, \omega) \chi_{\mathrm{eff}, 2}^{0}(q, \Delta \mathfrak{m}, \omega) \\
& \times V^{0}\left(q, \Delta \mathfrak{m}, r_{1}, r_{2}\right) V^{0}\left(q, \Delta \mathfrak{m}, r_{2}, r_{1}\right), \quad(\mathrm{B}
\end{aligned}
$$

where we have neglected the reciprocal lattice vectors different from zero and therefore used

$$
\begin{aligned}
& V^{0}\left(q_{i_{1}}, \Delta \mathfrak{m}_{i_{1}}, r_{1}, r_{2}\right) \mathcal{W}_{22}^{0}\left(q_{i_{1}}, \Delta \mathfrak{m}_{i_{1}}, q_{i_{2}}, \Delta \mathfrak{m}_{i_{2}}\right) \\
& \quad-V^{0}\left(q_{i_{1}}, \Delta \mathfrak{m}_{i_{1}}, r_{2}, r_{2}\right) \mathcal{W}_{12}^{0}\left(q_{i_{1}}, \Delta \mathfrak{m}_{i_{1}}, q_{i_{2}}, \Delta \mathfrak{m}_{i_{2}}\right) \simeq 0 .
\end{aligned}
$$

If we consider armchair-like tubes [only the linear bands from Eq. (A17)], then all the crystal angular momentum is zero and from the $g$-factor analysis in Sec. III A the interband transition $\left[\Pi=1 \leftrightarrow \Pi^{\prime}=-1\right.$ in Eq. (A17) $]$ can safely be neglected and for the intraband transition we have $g \sim 1$. Therefore 


$$
\begin{aligned}
\chi_{\mathrm{eff}, i}^{0}(q, 0, \omega) & =\frac{2}{2 \pi L} \sum_{k} \sum_{\Pi= \pm 1} \widetilde{\chi}_{i}^{0}(k \Pi, k+q \Pi, \omega) \\
& \equiv \chi_{\mathrm{eff}, i}^{0}(q, \omega)^{\Pi=+1}+\chi_{\mathrm{eff}, i}^{0}(q, \omega)^{\Pi=-1},
\end{aligned}
$$

and for $0 \leqslant q \leqslant \pi /|\mathbf{T}|$ we find in the long tube limit and for zero temperature $(T=0)$

$$
\begin{aligned}
\chi_{\text {eff }, i}^{0}(q, \omega)^{\Pi=+1} & \frac{2}{(2 \pi)^{2}}\left[\theta\left(k_{0}-\frac{\varepsilon_{\mathrm{F}}}{\hbar v_{0}}-q\right) \frac{v_{0} q\left(k_{0}+2 \varepsilon_{\mathrm{F}} / \hbar v_{0}\right)}{\omega^{2}-v_{0}^{2} q^{2}}\right. \\
& +\theta\left(q-k_{0}+\frac{\varepsilon_{\mathrm{F}}}{\hbar v_{0}}\right)\left\{\frac{2 v_{0} q(q-\pi /|\mathbf{T}|)}{v_{0}^{2} q^{2}-\omega^{2}}\right. \\
& \left.+\frac{1}{2 v_{0}} \ln \left(\left|\frac{\omega^{2}-v_{0}^{2}\left(q-2 k_{0}+2 \varepsilon_{\mathrm{F}} / \hbar v_{0}\right)^{2}}{\omega^{2}-v_{0}^{2} q^{2}}\right|\right)\right\} \\
& +\theta\left(q-\frac{1}{2} k_{0}-\frac{\varepsilon_{\mathrm{F}}}{\hbar v_{0}}\right) \frac{1}{2 v_{0}} \\
& \times \ln \left(\left|\frac{\omega^{2}-v_{0}^{2}\left(q-k_{0}-2 \varepsilon_{\mathrm{F}} / \hbar v_{0}\right)^{2}}{\omega^{2}-v_{0}^{2} q^{2}}\right|\right) \\
& \left.+\theta\left(\frac{1}{2} k_{0}+\frac{\varepsilon_{\mathrm{F}}}{\hbar v_{0}}-q\right) \frac{2 v_{0} q\left(\frac{1}{2} k_{0}+\varepsilon_{\mathrm{F}} / \hbar v_{0}-q\right)}{v_{0}^{2} q^{2}-\omega^{2}}\right]
\end{aligned}
$$

and

$$
\begin{aligned}
\chi_{\mathrm{eff}, i}^{0}(q, \omega)^{\Pi=-1} & \frac{2}{(2 \pi)^{2} \hbar}\left[\theta\left(k_{0}+\frac{\varepsilon_{\mathrm{F}}}{\hbar v_{0}}-q\right) \frac{2 v_{0} q\left(k_{0}+\varepsilon_{\mathrm{F}} / \hbar v_{0}-q\right)}{v_{0}^{2} q^{2}-\omega^{2}}\right. \\
& +\theta\left(q-k_{0}-\frac{\varepsilon_{\mathrm{F}}}{v_{0}}\right) \frac{1}{2 v_{0}} \\
& \times \ln \left(\left|\frac{v_{0}^{2}\left(q-2 k_{0}-2 \varepsilon_{\mathrm{F}} / \hbar v_{0}\right)^{2}-\omega^{2}}{v_{0}^{2} q^{2}-\omega^{2}}\right|\right) \\
& +\theta\left(\frac{1}{2} k_{0}-\frac{\varepsilon_{\mathrm{F}}}{\hbar v_{0}}-q\right) \frac{2 v_{0} q\left(k_{0}+\varepsilon_{\mathrm{F}} / \hbar v_{0}\right)}{\omega^{2}-v_{0}^{2} q^{2}}
\end{aligned}
$$

$$
\begin{aligned}
& +\theta\left(q-\frac{1}{2} k_{0}+\frac{\varepsilon_{\mathrm{F}}}{\hbar v_{0}}\right)\left\{\frac{2 v_{0} q(\pi /|\mathbf{T}|-q)}{\omega^{2}-v_{0}^{2} q^{2}}\right. \\
& \left.\left.+\frac{1}{2 v_{0}} \ln \left(\left|\frac{v_{0}^{2}\left(q-k_{0}+2 \varepsilon_{\mathrm{F}} / \hbar v_{0}\right)^{2}-\omega^{2}}{v_{0}^{2} q^{2}-\omega^{2}}\right|\right)\right\}\right],
\end{aligned}
$$

which for small $q$ and $\omega$ simplifies to the result in ${ }^{112}$

$$
\chi_{\mathrm{eff}, i}^{0}(q, \omega)^{\Pi=+1}=\chi_{\mathrm{eff}, i}^{0}(q, \omega)^{\Pi=-1}=\frac{4 v_{0} q^{2}}{(2 \pi)^{2} \hbar\left(\omega^{2}-\left(v_{0} q\right)^{2}\right)} .
$$

Note that in the static limit the effective polarizability is just a constant. The zero temperature approximation ${ }^{55}$ of the polarizability is good as long as $T$ is much smaller than $T_{\mathrm{F}}$, which is often the case for nanotubes $\left(T_{\mathrm{F}} \sim 1000 \mathrm{~K}\right)$. Including finite temperature in the polarizability could give a plasmon enhanced drag as previously found for bilayer systems $^{56,57}$ at $T \simeq 0.5 T_{\mathrm{F}}$. For zigzaglike tubes the effective polarizability can be found in the same way, but for the linear bands crossing the Fermi level $\left(\varepsilon_{\mathrm{F}}=0\right)$ we can-in contrast to the armchairlike case-have both $\Delta \mathfrak{m}=0$ and $\Delta \mathfrak{m}= \pm\left(\mathfrak{m}_{a}\right.$ $\left.-\mathfrak{m}_{b}\right)$.

The unscreened Coulomb interaction $V^{0}\left(q, \Delta \mathfrak{m}, r_{i}, r_{j}\right)$ can be found from the Poisson equation by Fourier transforming in the cylindrical coordinate and in the coordinate along the tube, i.e., ${ }^{80}$

$$
V^{0}\left(q, \Delta \mathfrak{m}, r_{i}, r_{j}\right)=\frac{e^{2}}{\epsilon_{0}} \mathrm{I}_{\Delta \mathfrak{m}}\left(q r_{i}\right) \mathrm{K}_{\Delta \mathfrak{m}}\left(q r_{j}\right), \quad r_{i} \leqslant r_{j},
$$

where $\mathrm{I}_{\Delta \mathfrak{m}}(x)\left[\mathrm{K}_{\Delta \mathfrak{m}}(x)\right]$ is the modified Bessel's functions of the first [second] kind of order $\Delta \mathfrak{m}$ and $\epsilon_{0}$ is the vacuum permittivity. Note that the small $q$ limit is (logarithmic) divergent only for the potential with $\Delta \mathfrak{m}=0$. So we have all the ingredients in the screened Coulomb matrix element between different shells using the tight-binding states of the carbon nanotubes, which is used to model the Coulomb drag between the shells.
*Email address: lunan@fys.ku.dk

${ }^{1}$ S. Reich, C. Thomsen, and J. Maultzsch, Carbon Nanotubes, 1st ed. (Wiley-VCH, Weinheim, 2003), for a nice and recent review.

${ }^{2}$ A. Bachtold, M. S. Fuhrer, S. Plyasunov, M. Forero, E. H. Anderson, A. Zettl, and P. L. McEuen, Phys. Rev. Lett. 84, 6082 (2000).

${ }^{3}$ W. Liang, M. Bockrath, D. Bozovic, J. H. Hafner, M. Tinkham, and H. Park, Nature (London) 441, 665 (2001).

${ }^{4}$ A. Bachtold, M. S. Fuhrer, S. Plyasunov, M. Forero, E. H. Anderson, A. Zettl, and P. L. McEuen, Phys. Rev. Lett. 84, 6082 (2000)

${ }^{5}$ A. Bachtold, C. Strunk, J.-P. Salvetat, J.-M. Bonard, L. Forró, T. Nussbaumer, and C. Schönenberger, Nature (London) 397, 673
(1999).

${ }^{6}$ C. Schönenberger, M. Buitelaar, M. Krüger, I. Widmer, T. Nussbaumer, and M. Iqbal, Proceedings Moriond 2001, cond-mat/ 0106501 (unpublished).

${ }^{7}$ R. Martel, T. Schmidt, H. R. Shea, T. Hertel, and P. Avouris, Appl. Phys. Lett. 73, 2447 (1998).

${ }^{8}$ S. Frank, P. Poncharal, Z. Wang, and W. A. de Heer, Science 280, 1744 (1998).

${ }^{9}$ M. Bockrath, D. H. Cobden, P. L. McEuen, N. G. Chopra, A. Zettl, A. Thess, and R. E. Smalley, Science 275, 1922 (1997).

${ }^{10}$ S. J. Tans, M. H. Devoret, H. Dai, A. Thess, R. E. Smalley, L. J. Geerligs, and C. Dekker, Nature (London) 386, 474 (1997).

${ }^{11}$ D. H. Cobden and J. Nygård, Phys. Rev. Lett. 89, 046803 (2002). 
${ }^{12}$ J. Nygård, D. H. Cobden, and P. E. Lindelof, Nature (London) 408, 342 (2000).

${ }^{13}$ P. Jarillo-Herrero, S. Sapmaz, C. Dekker, L. P. Kouwenhoven, and H. S. J. van der Zant, Nature (London) 429, 389 (2004).

${ }^{14}$ J. Nygård, D. H. Cobden, M. Bockrath, P. L. McEuen, and P. E. Lindelof, Appl. Phys. A: Mater. Sci. Process. 69, 297 (1999).

${ }^{15}$ P. L. McEuen, M. Bockrath, D. H. Cobden, Y.-G. Yoon, and S. G. Louie, Phys. Rev. Lett. 83, 5098 (1999).

${ }^{16}$ J. Kong, E. Yenilmez, T. W. Tombler, W. Kim, H. Dai, R. B. Laughlin, L. Liu, C. S. Jayanthi, and S. Y. Wu, Phys. Rev. Lett. 87, 106801 (2001).

${ }^{17}$ A. Javey, J. Guo, Q. Wang, M. Lundstrom, and H. Dai, Nature (London) 424, 654 (2003).

${ }^{18}$ D. Mann, A. Javey, J. Kong, Q. Wang, and H. Dai, Nano Lett. 3, 1541 (2003).

${ }^{19}$ R. Egger and A. O. Gogolin, Phys. Rev. Lett. 79, 5082 (1997).

${ }^{20}$ R. Egger and A. O. Gogolin, Eur. Phys. J. B 3, 281 (1998).

${ }^{21}$ M. Bockrath, D. H. Cobden, J. Lu, A. G. Rinzler, R. E. Smalley, L. Balents, and P. L. McEuen, Nature (London) 397, 598 (1999)

${ }^{22}$ H. Ishii et al., Nature (London) 426, 540 (2003).

${ }^{23}$ A. Kasumov, M. Kociak, M. Ferrier, R. Deblock, S. Guéron, B. Reulet, I. Khodos, O. Stéphan, and H. Bouchiat, Phys. Rev. B 68, 214521 (2003).

${ }^{24}$ R. Tarkiainen, M. Ahlskog, J. Penttilä, L. Roschier, P. Hakonen, M. Paalanen, and E. Sonin, Phys. Rev. B 64, 195412 (2001), and references therein.

${ }^{25}$ V. Krstić, S. Blumentritt, J. Muster, S. Roth, and A. Rubio, Phys. Rev. B 67, 041401(R) (2003).

${ }^{26}$ N. Kang, L. Lu, W. J. Kong, J. S. Hu, W. Yi, Y. P. Wang, D. L. Zhang, Z. W. Pan, and S. S. Xie, Phys. Rev. B 67, 033404 (2003).

${ }^{27}$ R. Egger, Phys. Rev. Lett. 83, 5547 (1999).

${ }^{28}$ T. Hunger, B. Lengeler, and J. Appenzeller, Phys. Rev. B 69, 195406 (2004).

${ }^{29}$ Y.-G. Yoon, P. Delaney, and S. G. Louie, Phys. Rev. B 66, 073407 (2002).

${ }^{30}$ A. A. Maarouf, C. L. Kane, and E. J. Mele, Phys. Rev. B 61, 11156 (2000), and reference therein.

${ }^{31}$ P. G. Collins, M. S. Arnold, and P. Avouris, Science 292, 706 (2001).

${ }^{32}$ J. Cumings, P. G. Collins, and A. Zettl, Nature (London) 406, 586 (2000).

${ }^{33}$ S. Dohn, master's thesis, Department of Micro and Nanotechnology, Technical University of Denmark, 2003.

${ }^{34}$ P. G. Collins and P. Avouris, Appl. Phys. A: Mater. Sci. Process. 74, 329 (2002).

${ }^{35}$ Q. H. Li and T. Wang, Sci. China, Ser. E: Technol. Sci. 47, 1 (2004).

${ }^{36}$ J. Cumings and A. Zettl, Science 289, 602 (2000).

${ }^{37}$ S. Roche, F. Triozon, A. Rubio, and D. Mayou, Phys. Rev. B 64, 121401(R) (2001).

${ }^{38}$ S. Roche, F. Triozon, A. Rubio, and D. Mayou, Phys. Lett. A 285, 94 (2001).

${ }^{39}$ F. Triozon, S. Roche, A. Rubio, and D. Mayou, Phys. Rev. B 69, 121410(R) (2004).

${ }^{40}$ K.-H. Ahn, Y.-H. Kim, J. Wiersig, and K. J. Chang, Physica E (Amsterdam) 22, 666 (2004).

${ }^{41}$ A. Hansson and S. Stafström, Phys. Rev. B 67, 075406 (2003).

${ }^{42}$ S. Sanvito, Y.-K. Kwon, D. Tománek, and C. J. Lambert, Phys.
Rev. Lett. 84, 1974 (2000).

${ }^{43}$ Y. Miyamoto, S. Saito, and D. Tománek, Phys. Rev. B 65, 041402(R) (2002).

${ }^{44}$ S. Uryu, Phys. Rev. B 69, 075402 (2004).

${ }^{45}$ D.-H. Kim and K. J. Chang, Phys. Rev. B 66, 155402 (2002).

${ }^{46}$ B. Bourlon, C. Miko, L. Forro, D. C. Glattli, and A. Bachtold, Phys. Rev. Lett. 93, 176806 (2004).

${ }^{47}$ M. B. Pogrebinskii, Fiz. Tekh. Poluprovodn. (S.-Peterburg) 11, 637 (1977) [Sov. Phys. Semicond. 11, 372 (1977)].

${ }^{48}$ P. J. Price, Physica B \& C 117, 750 (1983).

${ }^{49}$ Other $e-e$ interactions such as phonon mediated interaction could give an important contribution as seen in bilayer systems (Ref. 58). This could be included in future studies.

${ }^{50}$ Q. Zheng and Q. Jiang, Phys. Rev. Lett. 88, 045503 (2002), and citations of this paper.

${ }^{51}$ J. Nygård, P. Bøgglid, and P. Hakonen (private communication).

${ }^{52}$ T. Sugai, H. Yoshida, T. Shimada, T. Okazaki, H. Shinohara, and S. Bandow, Nano Lett. 3, 769 (2003).

${ }^{53}$ A. G. Rojo, J. Phys.: Condens. Matter 11, R31 (1999), for a good review.

${ }^{54}$ T. J. Gramila, J. P. Eisenstein, A. H. MacDonald, L. N. Pfeiffer, and K. W. West, Phys. Rev. Lett. 66, 1216 (1991).

${ }^{55}$ A.-P. Jauho and H. Smith, Phys. Rev. B 47, 4420 (1993).

${ }^{56}$ K. Flensberg and B. Y.-K. Hu, Phys. Rev. Lett. 73, 3572 (1994).

${ }^{57}$ N. P. R. Hill, J. T. Nicholls, E. H. Linfield, M. Pepper, D. A. Ritchie, G. A. C. Jones, B. Y.-K. Hu, and K. Flensberg, Phys. Rev. Lett. 78, 2204 (1997).

${ }^{58}$ M. C. Bønsager, K. Flensberg, B. Y.-K. Hu, and A. H. MacDonald, Phys. Rev. B 57, 7085 (1998).

${ }^{59}$ I. Gornyi, A. Mirlin, and F. von Oppen, Phys. Rev. B 70, 245302 (2004).

${ }^{60}$ A. Komnik and R. Egger, Phys. Rev. Lett. 80, 2881 (1998).

${ }^{61}$ K. Flensberg, Phys. Rev. Lett. 81, 184 (1998).

${ }^{62}$ Y. V. Nazarov and D. V. Averin, Phys. Rev. Lett. 81, 653 (1998).

${ }^{63}$ V. V. Ponomarenko and D. V. Averin, Phys. Rev. Lett. 85, 4928 (2000).

${ }^{64}$ R. Klesse and A. Stern, Phys. Rev. B 62, 16912 (2000).

${ }^{65}$ A. Komnik and R. Egger, Eur. Phys. J. B 19, 271 (2000).

${ }^{66}$ B. Trauzettel, R. Egger, and H. Grabert, Phys. Rev. Lett. 88, 116401 (2002).

${ }^{67}$ B. Gao, A. Komnik, R. Egger, D. C. Glattli, and A. Bachtold, Phys. Rev. Lett. 92, 216804 (2004).

${ }^{68}$ T. Ando and T. Nakanishi, J. Phys. Soc. Jpn. 67, 1704 (1998).

${ }^{69}$ T. Ando, T. Nakanishi, and R. Saito, J. Phys. Soc. Jpn. 67, 2857 (1998).

${ }^{70}$ R. Klesse, Phys. Rev. B 66, 085409 (2002).

${ }^{71}$ C. T. White, D. H. Robertson, and J. W. Mintmire, Phys. Rev. B 47, 5485 (1993).

${ }^{72}$ J. W. Mintmire and C. T. White, Carbon 33, 893 (1995).

${ }^{73}$ T. Vuković, I. Milošević, and M. Damnjanović, Phys. Rev. B 65, 045418 (2002).

${ }^{74}$ M. Damnjanović, I. Milošević, T. Vuković, and R. Sredanović, Phys. Rev. B 60, 2728 (1999).

${ }^{75} \mathrm{We}$ do not have exchange interaction between the tubes, since the inter tube tunnelling were neglected. Therefore we can use product states instead of antisymmetrized wave functions.

${ }^{76}$ R. S. Lee, H. J. Kim, J. E. Fischer, J. Lefebvre, M. Radosavljević, J. Hone, and A. T. Johnson, Phys. Rev. B 61, 4526 (2000).

${ }^{77}$ C. Zhou, J. Kong, E. Yenilmez, and H. Dai, Science 290, 1552 (2000). 
${ }^{78}$ Note that in doing the wrapping of the graphene band structure we are going from a two- to three-dimensional description and thereby the radial part of the wave function is $|\psi| \propto \delta\left(r-r^{c}\right) / r$, where $r^{c}$ is the radius of the tube.

${ }^{79}$ The matrix elements (11) and (12) could also have been obtained using the helical symmetry states $|\kappa \mathfrak{m}\rangle$, which has the advantage of making the $g$ factor an explicitly periodic function of reciprocal lattice vectors, whereas using the translational unit cell this periodicity has to be imposed by hand (Ref. 80). The states are given explicitly in Refs. 71 and 72.

${ }^{80}$ A. M. Lunde, master's thesis, Niels Bohr Institute and Department of Micro and Nanotechnology, University of Copenhagen, see www.fys.ku.dk/flensberg/students.htm (2004).

${ }^{81}$ K. Flensberg and B. Y.-K. Hu, Phys. Rev. B 52, 14796 (1995).

${ }^{82}$ A. Alkauskas, K. Flensberg, B. Y.-K. Hu, and A.-P. Jauho, Phys. Rev. B 66, 201304(R) (2002).

${ }^{83}$ B. Y.-K. Hu, Phys. Rev. B 57, 12345 (1998).

${ }^{84}$ K. Flensberg, B. Y.-K. Hu, A.-P. Jauho, and J. M. Kinaret, Phys. Rev. B 52, 14761 (1995).

${ }^{85} \mathrm{H}$. Smith and H. H. Jensen, Transport Phenomena (Clarendon Press, Oxford, 1989).

${ }^{86}$ M. Pustilnik, E. G. Mishchenko, L. I. Glazman, and A. V. Andreev, Phys. Rev. Lett. 91, 126805 (2003).

${ }^{87}$ N. A. Mortensen, K. Flensberg, and A.-P. Jauho, Phys. Rev. Lett. 86, 1841 (2001).

${ }^{88}$ N. A. Mortensen, K. Flensberg, and A.-P. Jauho, Phys. Rev. B 65 , 085315 (2002).

${ }^{89}$ V. L. Gurevich, V. B. Pevzner, and E. W. Fenton, J. Phys.: Condens. Matter 10, 2551 (1998).

${ }^{90}$ The definition of electron-hole symmetry can be given as follows: For each occupied $k$ state (at $T=0$ ) with energy $\varepsilon_{k}$ and velocity $v_{k}$ there exists one and only one empty state $k^{\prime}$ with the same velocity $v_{k^{\prime}}=v_{k}$ and the opposite energy with respect to the Fermi level: $\varepsilon_{\mathrm{F}}-\varepsilon_{k^{\prime}}=-\left(\varepsilon_{\mathrm{F}}-\varepsilon_{k}\right)$. In this way there is a one to one correspondence between the electrons (i.e., the filled states) and holes (i.e., the empty states).

${ }^{91}$ M. Krüger, M. R. Buitelaar, T. Nussbaumer, C. Schönenberger, and L. Forró, Appl. Phys. Lett. 78, 1291 (2001).

${ }^{92}$ C. Zhou, J. Kong, and H. Dai, Phys. Rev. Lett. 84, 5604 (2000).

${ }^{93}$ A. Javey, H. Kim, M. Brink, Q. Wang, A. Ural, J. Guo, P. Mcintyre, P. Mceuen, M. Lundstrom, and H. Dai, Nat. Mater. 1, 241
(2002).

${ }^{94}$ A. M. Lunde and A.-P. Jauho, Semicond. Sci. Technol. 19, S433 (2004).

${ }^{95}$ B. Y.-K. Hu and K. Flensberg, Hot Carriers in Semiconductors (HCIS-9), edited by K. Hess et al. (Plenum Press, Chicago, 1996), p. 421.

${ }^{96}$ In Ref. $20 \epsilon_{r}=1.4$ is used.

${ }^{97}$ Note that the crystal angular momentum conservation depend on wether the tubes are commensurable or not (Ref. 80).

${ }^{98}$ G. Qin, J. Phys.: Condens. Matter 7, 9785 (1995).

${ }^{99}$ N. Hamada, S.-I. Sawada, and A. Oshiyama, Phys. Rev. Lett. 68, 1579 (1992).

${ }^{100}$ R. Saito, M. Fujita, G. Dresselhaus, and M. S. Dresselhaus, Phys. Rev. B 46, 1804 (1992).

${ }^{101}$ R. Saito, G. Dresselhaus, and M. S. Dresselhaus, Physical Properties of Carbon Nanotubes, 3rd ed. (Imperial College Press, London, 1998).

${ }^{102}$ P. R. Wallace, Phys. Rev. 71, 622 (1947).

${ }^{103}$ N. Ashcroft and N. Mermin, Solid State Physics, college ed. (Harcourt College Publishers, Orlando, 1976).

${ }^{104}$ S. D. M. Brown, P. Corio, A. Marucci, M. A. Pimenta, M. S. Dresselhaus, and G. Dresselhaus, Phys. Rev. B 61, 7734 (2000).

${ }^{105}$ S. Reich, J. Maultzsch, C. Thomsen, and P. Ordejón, Phys. Rev. B 66, 035412 (2002).

${ }^{106}$ J. W. G. Wilder, L. C. Venema, A. G. Rinzler, R. E. Smalley, and C. Dekker, Nature (London) 391, 59 (1998).

${ }^{107}$ T. W. Odom, J.-L. Huang, P. Kim, and C. M. Lieber, Nature (London) 391, 62 (1998).

${ }^{108}$ To get a feeling for the connection between the description of the band structure using the translational and primitive unit cell one can use the MATHEMATICA program: atomic and electronic structure of carbon nanotubes, by M. Brandbyge, found at http:// library.wolfram.com/infocenter/MathSource/384/.

${ }^{109}$ Note that graphene has six zeros all at the FBZ boundary and these are given in three pairs connected by reciprocal lattice vectors of graphene.

${ }^{110} \mathrm{We}$ only have numerical proof for this property, found by trying all combinations of $n, m \leqslant 100$.

${ }^{111}$ H. Ajiki and T. Ando, J. Phys. Soc. Jpn. 62, 1255 (1993).

${ }^{112}$ W. Que, Phys. Rev. B 66, 193405 (2002). 